\title{
Study of the Fractional-Order HIV-1 Infection Model with Uncertainty in Initial Data
}

\author{
Yong Wu, ${ }^{1}$ Shabir Ahmad $\mathbb{D}^{2},{ }^{2}$ Aman Ullah $\mathbb{D}^{2},{ }^{2}$ and Kamal Shah $\mathbb{D}^{2}$ \\ ${ }^{1}$ School of Tourism Date, Guilin Tourism University, Guilin 541006, China \\ ${ }^{2}$ Department of Mathematics, University of Malakand, Chakdara, Dir Lower, Khyber Pakhtunkhwa, Pakistan
}

Correspondence should be addressed to Kamal Shah; kamalshah408@gmail.com

Received 28 October 2021; Revised 14 December 2021; Accepted 4 January 2022; Published 21 January 2022

Academic Editor: Nasser Hassen Sweilam

Copyright (c) 2022 Yong Wu et al. This is an open access article distributed under the Creative Commons Attribution License, which permits unrestricted use, distribution, and reproduction in any medium, provided the original work is properly cited.

Uncertainty always lives with us. We cannot take exact measurement of initial conditions or parameters values in a mathematical model. As humans, we are remaining alive in an environment where the uncertainties lie in the modelling of physical phenomena. There might be some incomplete information or estimation of the parameter or initial values. To handle uncertainty, we use fuzzy operators rather than classical operators. In this paper, we study a model of HIV-1 infection by taking uncertainty in the initial data under Caputo fractional operator. We explore the existence and uniqueness of the results through fixed-point theory. We study the Ulam-Hyres stability of the considered model. By using the fuzzy Laplace Adomian decomposition method, numerical results are obtained for specific fuzzy initial conditions. To better understand the behaviour of the fuzzy solution, we present the obtained numerical results graphically for various fractional orders where the uncertainty lies in $[0,1]$.

\section{Introduction}

AIDS (acquired immune deficiency syndrome) is one of the most dangerous diseases caused by a pathogen virus called HIV (human immunodeficiency virus) and leads a person to death. In 1982, a patient in the USA was infected by HIV. At the end of 2019, about 38 million people, identified by the WHO (World Health Organization), were surviving with HIV. In 2019, 0.69 million people died from diseases associated with AIDS. To investigate how AIDS can spread or control, mathematical models are valuable instruments as they provide a short or a long-term prediction of the frequency of the disease. Many researchers have investigated the dynamics of HIV infection models [1-3]. A lot of methods have been used by researchers to find a solution to HIV infections [4-6]. In [7], the authors presented an HIV-1 infection model which contains five compartments, denoted by $\check{M}(t)$ (the uninfected CD4+T cells), $\check{M}^{*}(t)$ (the concentration of infected cells), $\check{M}^{* *}(t)$ (the concentration of double cells), and $\mathbb{V}_{p}(t)$ and $\mathbb{V}_{r}(t)$ (the densities of pathogen viruses), respectively. The integer-order model of HIV-1 infection is given by

$$
\left\{\begin{array}{l}
\frac{d}{d t} \check{\mathbb{M}}(t)=\lambda-d_{1} \check{\mathscr{M}}(t)-\beta_{1} \check{M}(t) \mathscr{V}_{p}(t), \\
\frac{d}{d t} \check{\mathbb{M}}^{*}(t)=\beta_{1} \check{\mathbb{M}}(t) \mathscr{V}_{p}(t)-d_{2} \check{M}^{*}(t)-\epsilon_{1} \mathscr{V}_{r}(t) \check{\mathbb{M}}^{*}(t), \\
\frac{d}{d t} \check{\mathbb{M}}^{* *}(t)=\epsilon_{1} \mathscr{V}_{r}(t) \check{\mathbb{M}}^{*}(t)-d_{3} \check{\mathbb{M}}^{* *}(t), \\
\frac{d}{d t} \mathbb{V}_{p}(t)=k^{\prime} \check{\mathbb{M}}^{*}(t)-d_{4} \mathbb{V}_{p}(t), \\
\frac{d}{d t} \mathbb{V}_{r}(t)=c^{\prime} \check{\mathbb{M}}^{* *}(t)-d_{5} \mathbb{V}_{r}(t),
\end{array}\right.
$$


where the host cells are produced at the rate $\lambda$. Moreover, $d_{1}, d_{2}, d_{3}$, and $d_{4}$ are the rates of death of host cells, infected cells, double infected cells, and pathogen virus, respectively. $\beta_{1}$ is the rate of infection of healthy CD4+ T cells. The rate of production of HIV- 1 by a cell is defined by $k^{\prime}, \epsilon_{1}$ is infection rate of removal of HIV-1 infected cells, $c^{\prime}$ is the rate of production of recombinant by a double-infected cell, and $d_{5}$ is the removal rate of recombinant. The mathematician has taken an interest in fractional calculus in recent years as it has many applications in engineering and biological sciences [8-10]. In the last decade, the researcher converted many models from integer order to fractional order which provides best results as compared to integer-order derivatives. As fractional operator has a greater degree of freedom, different researchers studied the existence theory of the solution [11-13]. The fractional operators also have been used for modelling of different infectious diseases. Sweilam et al. investigated the optimal control for variable order fractional HIV/AIDS and malaria mathematical models with multitime delay [14]. The Legendre spectral-collocation method was used for solving fractional optimal control of HIV infection of CD4+T cells' mathematical model in [15]. A complex fractional-order HIV infection model with drug resistance during therapy has been studied by Sweilam et al. [16]. Some other applications of the fractional-order operators in various field of sciences can be found in [17-19]. It is worthy to note here that the fractional derivative in the Caputo sense is the most commonly used definition among the definitions of the fractional derivative. The definition of Caputo is mathematically rigorous than the Riemann-Liouville definition. The Caputo derivative exists in the whole interval $(0,1)$. In addition, Caputo definition is very welcome in applied science and engineering. Furthermore, properties of the Caputo derivative are helpful in translating the higher fractional-order differential systems into lower ones. For a comparison between Caputo and Riemann-Liouville operators, the interested reader is referred to $[20,21]$. Therefore, we will consider the fractional operator in Caputo sense.

Confusion and uncertainty still live with us, and that is a reality. Many human beings are exposed to doubting anything surrounding them and questioning why for themselves or others. Because they are not clear, and their reports are incomplete or inaccurate. Now, suppose that we are in a situation where uncertainty occurs with all this inaccurate knowledge. It is a reality that, in many of our real questions where inaccurate data are involved, we do not know how to answer. For scientists, this mindset and attitude of uncertainty is very necessary. Our aim is to sort out how to understand it and operate through it rather than trying to fight ambiguity because the advancement, resources, and life that you want are uncertain. To overcome this situation, Zadeh introduced fuzzy set in 1965 [22]. The concept of fuzzy mapping and control has been addressed by Zadeh and Chang [23]. Furthermore, researchers extended the ordinary and fractional operators to fuzzy operators $[24,25]$. These operators have been used by various authors to study different models [26-29]. If the information or data is imprecise, then fuzzy operators model physical phenomena easily and accurately. We are interested in investigating model (1) for fractional-order derivative under the uncertain initial conditions. For fractional order $0<\gamma \leq 1$, consider

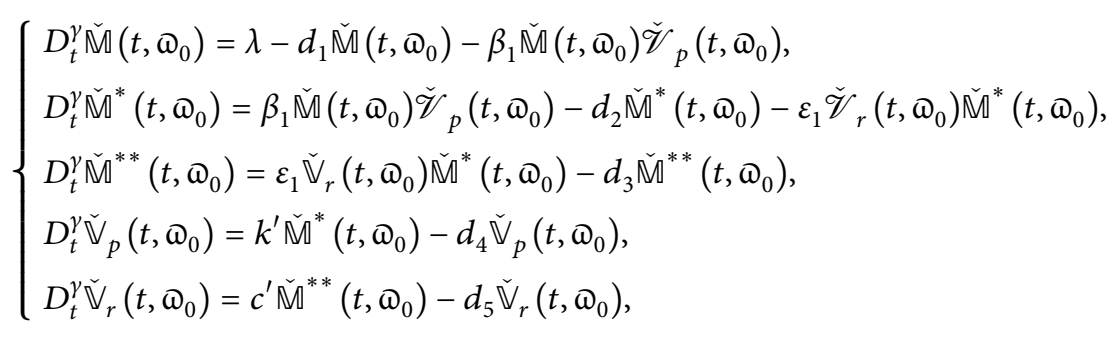

along with fuzzy initial conditions,

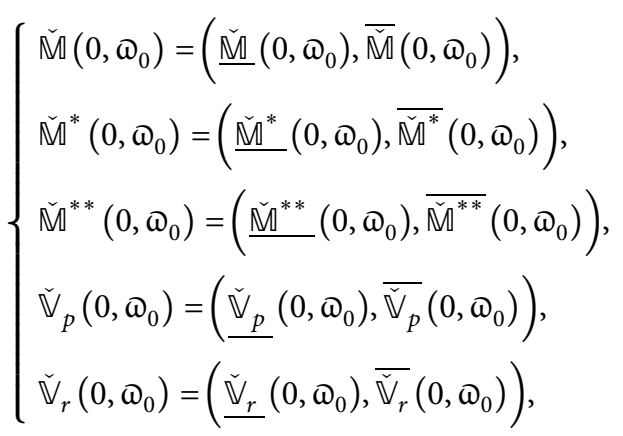

such that $\varpi_{0} \in[0,1]$.

\section{Preliminaries}

This section provides the basic concepts of fuzzy sets and fuzzy fractional calculus. Let FFD, FFI, and LT denote the fuzzy fractional derivative, fuzzy fractional integral, and Laplace transform, respectively.

Definition 1 (see $[29,30]$ ). A fuzzy set $\tilde{d}: \mathbb{R} \longrightarrow[0,1]$ is called a fuzzy number if

(i) $\tilde{d}$ is fuzzy convex.

(ii) $\tilde{d}$ is normal, i.e., for some $\left(z_{0} \in \mathbb{R} ; \tilde{d}\left(z_{0}\right)=1\right)$.

(iii) $\dot{\tilde{d}}$ is upper semicontinuous on $\mathbb{R}$. 
(iv) The closure of $\{z \in \mathbb{R}, \tilde{d}(z)>0\}$ is compact.

Definition 2 (see $[29,30])$. The parametric form of a fuzzy number $\widetilde{d}$ is given by $\left[\underline{\tilde{d}}\left(u, \omega_{0}\right), \widetilde{d}\left(u, \omega_{0}\right)\right]$, for $\omega_{0} \in[0,1]$, if and only if

(i) $\underline{\tilde{d}}\left(u, \Phi_{0}\right)$ is increasing, left on $[0,1]$ and right continuous at 0 , respectively.

(ii) $\overline{\widetilde{d}}\left(u, \omega_{0}\right)$ is decreasing, left on $[0,1]$ and right continuous at 0 , respectively.

(iii) $\underline{\tilde{d}}\left(u, \omega_{0}\right) \leq \overline{\widetilde{d}}\left(u, \omega_{0}\right)$.

Definition 3 (see [31]). Let $\mathfrak{\Im}: E \times E \longrightarrow \mathbb{R}$ be a function, and $b=\left(\underline{b}\left(\Phi_{0}\right), \bar{b}\left(\Phi_{0}\right)\right)$ and $c=\left(\underline{c}\left(\Phi_{0}\right), \bar{c}\left(\Phi_{0}\right)\right)$ are any two fuzzy numbers. Then, the $\mathrm{H}$-distance between $b$ and $c$ is given by

$\mathfrak{J}(b, c)=\sup _{\Phi_{0} \in[0,1]}\left[\max \left\{\left|\underline{b}\left(\omega_{0}\right)-\underline{c}\left(\varpi_{0}\right)\right|,\left|\bar{b}\left(\omega_{0}\right)-\bar{c}\left(\omega_{0}\right)\right|\right\}\right]$.

In $E, \mathfrak{I}$ satisfies the properties given below:

(i) $\mathfrak{I}(b+v, c+v)=\mathfrak{I}(b, c) \forall b, v, c \in E$.

(ii) $\mathfrak{\Im}(b\rangle, c\rangle)=|\rangle|\mathfrak{I}(b, c) \forall\rangle \in \mathbb{R}$ and $b, c \in E$.

(iii) $\mathfrak{I}(b+\xi, c+\varsigma) \leq \mathfrak{J}(b, c)+\mathfrak{I}(\xi, \varsigma) \forall b, c, \xi, \varsigma \in E$.

(iv) $(E, \mathfrak{I})$ is a complete metric space.

Definition 4 (see [31]). Let $\Lambda$ be a continuous fuzzy function on $[0, d] \subseteq \mathbb{R}$, and a FFI is defined as

$$
I^{\kappa} \Lambda(t)=\frac{1}{\Gamma(\gamma)} \int_{0}^{t}(t-\zeta)^{\gamma-1} \Lambda(\zeta) d \zeta .
$$

Furthermore, if $C^{\mathscr{R}_{\mathbb{F}}}[0, b] \cap L^{\mathscr{R}_{\mathbb{F}}}[0, b]$, where $C^{\mathscr{R}_{\mathbb{F}}}[0, b]$ and $L^{\mathscr{R}_{\mathbb{F}}}[0, b]$ are the spaces of fuzzy continuous functions and fuzzy Lebesgue integrable functions, respectively, then the FFI can be written as

$$
\left[I^{\gamma} \Lambda(t)\right]_{{\varpi_{0}}}=\left[I^{\gamma} \underline{\Lambda}_{\Theta_{0}}(t), I^{\gamma} \bar{\Lambda}_{\varpi_{0}}(t)\right], 0 \leq{\varpi_{0}} \leq 1,
$$

where

$$
\begin{aligned}
& I^{\gamma} \underline{\Lambda}_{\Theta_{0}}(t)=\frac{1}{\Gamma(\gamma)} \int_{0}^{t}(t-\zeta)^{\gamma-1} \underline{\Lambda}_{\Theta_{0}}(t) d \zeta, \\
& I^{\gamma} \bar{\Lambda}_{\varpi_{0}}(t)=\frac{1}{\Gamma(\gamma)} \int_{0}^{t}(t-\zeta)^{\gamma-1} \bar{\Lambda}_{\varpi_{0}}(t) d \zeta .
\end{aligned}
$$

Definition 5 (see [31]). A fuzzy function $\Lambda \in C^{\mathscr{R}_{\mathbb{F}}}[0, b] \cap L^{\mathscr{R}_{\mathbb{F}}}[0, b]$ is such that $\Lambda=\left[\underline{\Lambda}_{\Theta_{0}}(t), \bar{\Lambda}_{\omega_{0}}(t)\right]$, $t_{1} \in(0, b)$; then, the FFD in Caputo sense is given by

$$
\left[D^{\gamma} \Lambda\left(t_{0}\right)\right]_{\Phi_{0}}=\left[D^{\gamma} \underline{\Lambda}_{\Theta_{0}}\left(t_{0}\right), D^{\gamma} \bar{\Lambda}_{\varpi_{0}}\left(t_{0}\right)\right],
$$

where

$$
\begin{aligned}
& D^{\gamma} \underline{\Lambda}_{\Theta_{0}}\left(t_{0}\right)=\frac{1}{\Gamma(n-\gamma)}\left[\int_{0}^{t}(t-\zeta)^{n-\gamma-1} \frac{d^{n}}{d \zeta^{n}} \underline{\Lambda}_{\Theta_{0}}(\zeta) d \zeta\right]_{t=t_{0}}, \\
& D^{\gamma} \bar{\Lambda}_{\Theta_{0}}\left(t_{0}\right)=\frac{1}{\Gamma(n-\gamma)}\left[\int_{0}^{t}(t-\zeta)^{n-\gamma-1} \frac{d^{n}}{d \zeta^{n}} \bar{\Lambda}_{\Theta_{0}}(\zeta) d \zeta\right]_{t=t_{0}},
\end{aligned}
$$

where $n=[\gamma]$.

Definition 6 (see [32]). Let $\tilde{\mathscr{V}} \in C^{\mathscr{R}_{\mathbb{F}}}[0, b] \cap L^{\mathscr{R}_{\mathbb{F}}}[0, b]$. Then, the fuzzy LT of $\widetilde{\Lambda}(t)$ is defined as

$$
\mathscr{L}[\tilde{\Lambda}(t)]=\int_{0}^{\infty} \tilde{\Lambda}(t) \exp (-s \mathrm{t}) d t
$$

While, in the parametric form, the fuzzy LT is given by

$$
\begin{array}{r}
\int_{0}^{\infty} \tilde{\Lambda}(t) \exp (-s \mathrm{t}) d t=\left[\int_{0}^{\infty} \underline{\tilde{\Lambda}}\left(t, \oplus_{0}\right) \exp (-s \mathrm{t}) d t, \int_{0}^{\infty} \overline{\widetilde{\Lambda}}\left(t, \oplus_{0}\right) \exp (-s \mathrm{t}) d t\right] . \\
L\left[\left(D^{\gamma} \mathfrak{\Im}(t)\right)\right]=s^{\gamma} L[\mathfrak{\Im}(t)]-s^{\gamma-1}[\mathfrak{I}(0)] .
\end{array}
$$

Hence,

$$
\mathscr{L}[\widetilde{\Lambda}(t)]=\left[\mathscr{L}\left[\underline{\tilde{\Lambda}}\left(t, \oplus_{0}\right)\right], \mathscr{L}\left[\overline{\widetilde{\Lambda}}\left(t, \oplus_{0}\right)\right]\right] .
$$

Theorem 1 (see [31]). The LT of Caputo fractional derivative is defined as

\section{Existence and Uniqueness Results}

In this section, the existence and uniqueness of the solution of the fuzzy fractional model via fixed-point theory are discussed. Let us write the right-hand side of system (2) as 


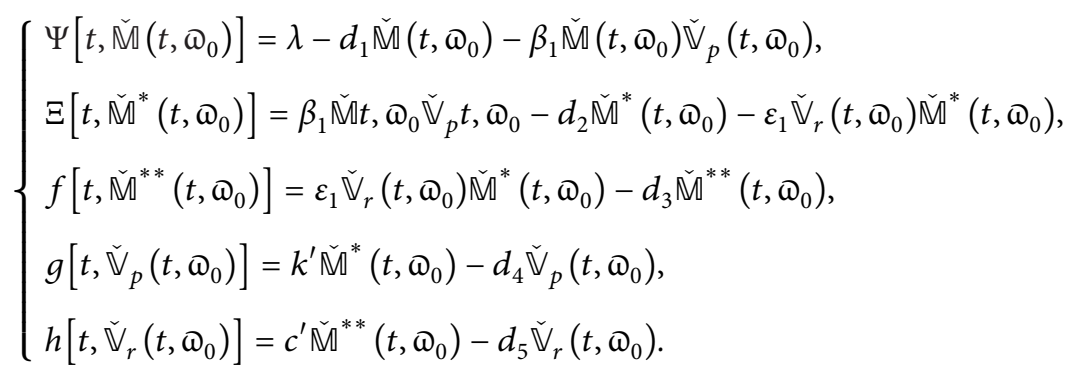

We can write the given system (2) as Now, using fractional integral, we obtain

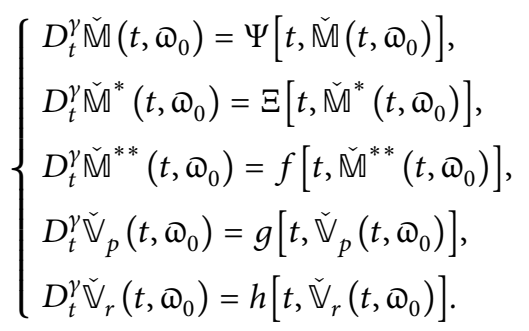

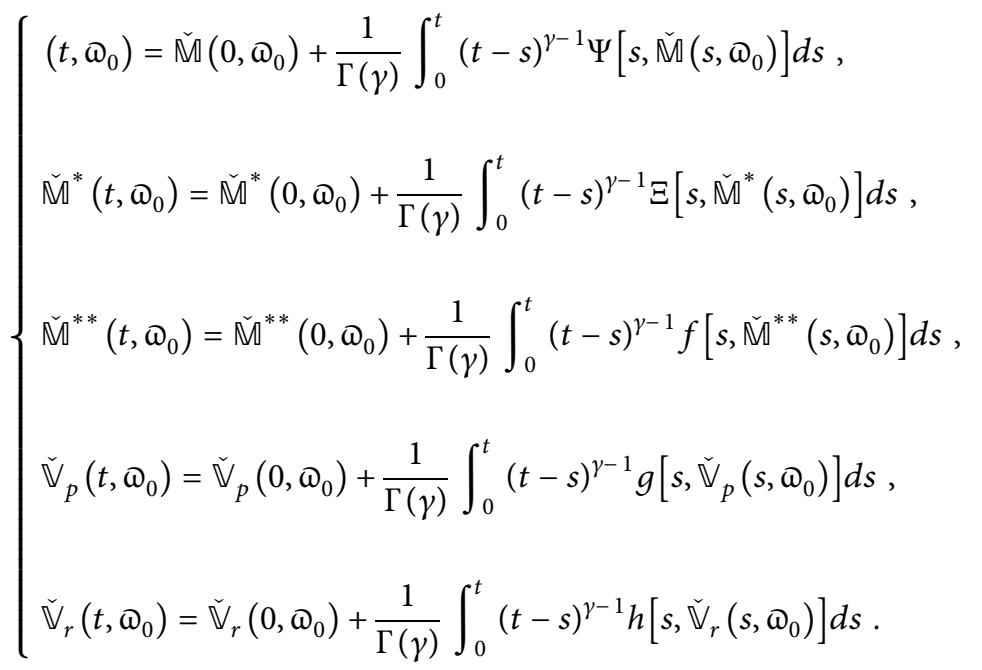

Now, consider a Banach space as $\mathbb{B}=\mathbb{B}_{1} \times \mathbb{B}_{2} \times$ $\mathbb{B}_{3} \times \mathbb{B}_{4} \times \mathbb{B}_{5}$, under the norm,

$$
\begin{aligned}
& \left\|\check{M}\left(t, \varpi_{0}\right), \check{M}^{*}\left(t, \varpi_{0}\right), \check{\mathbb{M}}^{* *}\left(t, \varpi_{0}\right), \check{\mathbb{V}}_{p}\left(t, \varpi_{0}\right), \check{\mathbb{V}}_{r}\left(t, \varpi_{0}\right)\right\| \\
& =\max _{t \in[0, T]}\left[\left|\check{M}\left(t, \varpi_{0}\right)+\check{\mathscr{M}}^{*}\left(t, \varpi_{0}\right)+\check{\mathbb{M}}^{* *}\left(t, \varpi_{0}\right),+\check{V}_{p}\left(t, \varpi_{0}\right)+\check{\mathbb{V}}_{r}\left(t, \varpi_{0}\right)\right|\right] \text {. }
\end{aligned}
$$

We can write equation (16) as 


$$
\check{\Pi}\left(t, \varpi_{0}\right)=\check{\Pi}\left(0, \varpi_{0}\right)+\frac{1}{\Gamma \gamma} \int_{0}^{t}(t-\Omega)^{\gamma-1} \Theta(\Lambda, \check{\Pi}(\Omega)) d \Omega,
$$

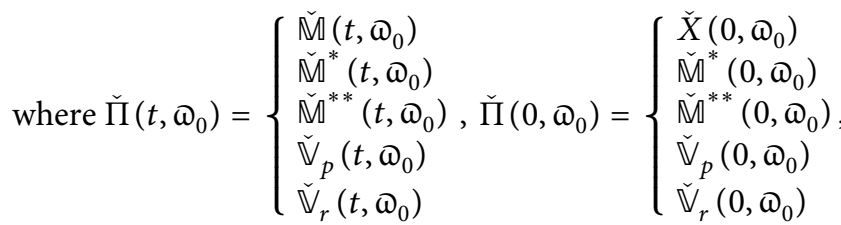

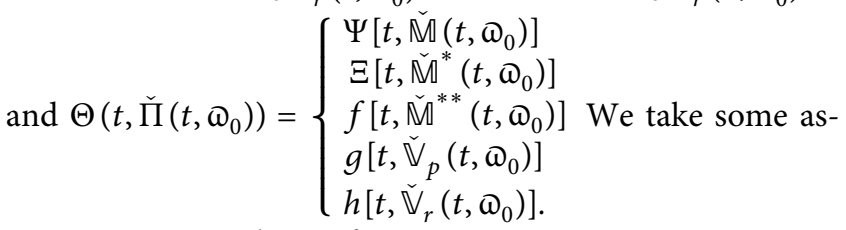
sumption on nonlinear function $\Theta: \mathbb{B} \longrightarrow \mathbb{B}$ as

(1) Lipschtiz condition: there exists a constant $K_{\check{\Pi}}>0$ such that, for each $\check{\Pi}_{1}\left(t, \varpi_{0}\right), \check{\Pi}_{2}\left(t, \varpi_{0}\right) \in \mathbb{B}$,

$$
\begin{gathered}
\left|\Theta\left(t, \check{\Pi}_{1}\left(t, \varpi_{0}\right)\right)-\Theta\left(t, \check{\Pi}_{2}\left(t, \varpi_{0}\right)\right)\right| \\
\leq K_{\check{\Pi}}\left|\check{\Pi}_{1}\left(t, \varpi_{0}\right)-\check{\Pi}_{2}\left(t, \varpi_{0}\right)\right| .
\end{gathered}
$$

(2) Growth condition: there exists constants $M_{\check{\Pi}}>0$ and $N_{\check{\Pi}}>0$ such that

$$
\left|\Theta\left(t, \check{\Pi}\left(t, \varpi_{0}\right)\right)\right| \leq M_{\check{\Pi}}\left|\check{\Pi}\left(t, \varpi_{0}\right)\right|+N_{\check{\Pi}} .
$$

Theorem 2. If the growth condition is satisfied, then system (3) has at least one solution.

Proof. Consider $\mathscr{A}=\left\{\check{\Pi}\left(t, \varpi_{0}\right) \in \mathbb{B}:\left\|\check{\Pi}\left(t, \varpi_{0}\right)\right\| \leq r\right\} \subset \mathbb{B}$ is closed and fuzzy convex, and a mapping $\psi: \mathscr{A} \longrightarrow A$ is defined by

$$
\begin{aligned}
\psi\left(\check{\Pi}\left(t, \oplus_{0}\right)\right)= & \check{\Pi}\left(0, \oplus_{0}\right) \\
& +\frac{1}{\Gamma(\gamma)} \int_{0}^{t}(t-\Omega)^{\gamma-1} \Theta\left(\Omega, \check{\Pi}\left(t, \oplus_{0}\right)\right) d \Omega .
\end{aligned}
$$

Then, for any $\check{\Pi}\left(t, \oplus_{0}\right) \in \mathscr{A}$, we have

$$
\begin{aligned}
& \left\|\psi\left(\check{\Pi}\left(t, \oplus_{0}\right)\right)\right\|=\max _{t \in[0, T]}\left|\check{\Pi}\left(0, \oplus_{0}\right)+\frac{1}{\Gamma(\gamma)} \int_{0}^{t}(t-\Omega)^{\gamma-1} \Theta\left(\Omega, \check{\Pi}\left(t, \oplus_{0}\right)\right) d \Omega\right|, \\
& \leq\left|\check{\Pi}\left(0, \varpi_{0}\right)\right|+\frac{1}{\Gamma(\gamma)} \int_{0}^{t}(t-\Omega)^{\gamma-1}\left|\Theta\left(\Omega, \check{\Pi}\left(t, \varpi_{0}\right)\right)\right| d \Omega, \\
& \leq\left|\check{\Pi}\left(0, \varpi_{0}\right)\right|+\frac{1}{\Gamma(\gamma)} \int_{0}^{t}(t-\Omega)^{\gamma-1}\left[M_{\Pi}\left|\check{\Pi}\left(t, \varpi_{0}\right)\right|+N_{\Pi}\right] d \Omega, \\
& \leq\left|\check{\Pi}\left(0, \varpi_{0}\right)\right|+\frac{\tau^{\gamma}}{\Gamma(\gamma+1)}\left[M_{\check{\Pi}}\left|\check{\Pi}\left(t, \varpi_{0}\right)\right|+N_{\check{\Pi}}\right] .
\end{aligned}
$$

It follows that $\left\|\psi\left(\check{\Pi}\left(t, \oplus_{0}\right)\right)\right\| \leq r$. Thus, $\psi(\mathscr{A}) \subset \mathscr{A}$; it implies that $\psi$ is bounded. To show $\psi$ is equi-continuity, consider $\phi_{1}, \phi_{2} \in[0, T]$ such that $\phi_{1}<\phi_{2}$. Then,

$$
\begin{aligned}
\left\|\psi\left(\check{\Pi}\left(t, \oplus_{0}\right)\right)\left(\phi_{2}\right)-\psi\left(\check{\Pi}\left(t, \oplus_{0}\right)\right)\left(\phi_{1}\right)\right\|= & \frac{1}{\Gamma(\gamma)} \int_{0}^{\phi_{2}}\left(\phi_{2}-\Omega\right)^{\gamma-1} \Theta\left(\Omega, \check{\Pi}\left(t, \oplus_{0}\right)\right) d \Omega \\
& -(\gamma) \int_{0}^{\phi_{1}}\left(\phi_{1}-\Omega\right)^{\gamma-1} \Theta\left(\Omega, \check{\Pi}\left(t, \oplus_{0}\right)\right) d \Omega, \\
\leq & {\left[\phi_{2}^{\gamma}-\phi_{1}^{\gamma}\right] \frac{\left[M_{\check{\Pi}}\left|\check{\Pi}\left(t, \oplus_{0}\right)\right|+N_{\check{\Pi}}\right]}{\Gamma(\gamma+1)} . }
\end{aligned}
$$

Hence,

$\left\|\psi\left(\check{\Pi}\left(t, \varpi_{0}\right)\right)\left(\phi_{2}\right)-\psi\left(\check{\Pi}\left(t, \varpi_{0}\right)\right)\left(\phi_{1}\right)\right\| \longrightarrow 0$ as $\phi_{2} \longrightarrow \phi_{1}$.
This show that the operator $\psi$ is equi-continuous, which is completely continuous using Arzela-Ascoli theorem, as $\psi$ is bounded. Therefore, system (3) has at least one solution using Schauder's fixed-point theorem. 
Theorem 3. Let the Lipschitz condition hold. If

$$
\tau^{\gamma} K_{\Pi}<\Gamma(\gamma+1),
$$

then system (3) has a unique solution.

Proof. Let $\check{\Pi}_{1}\left(t, \varpi_{0}\right), \check{\Pi}_{2}\left(t, \varpi_{0}\right) \in \mathbb{B}$. Then,

$$
\begin{aligned}
\left\|\psi\left(\check{\Pi}_{1}\left(t, \oplus_{0}\right)\right)-\psi\left(\check{\Pi}_{2}\left(t, \varpi_{0}\right)\right)\right\|= & \max _{t \in[0, T]} \frac{1}{\Gamma \gamma} \int_{0}^{t}(t-\Omega)^{\gamma-1} \Theta\left(\Omega, \check{\Pi}_{1}\left(t, \oplus_{0}\right)\right) d \Omega \\
& -\frac{1}{\Gamma \gamma} \int_{0}^{t}(t-\Omega)^{\gamma-1} \Theta\left(\Omega, \check{\Pi}_{2}\left(t, \varpi_{0}\right)\right) d \Omega, \\
\leq & \frac{\tau^{\gamma}}{\Gamma(\gamma+1)} K_{\check{\Pi}}\left|\check{\Pi}_{1}\left(t, \oplus_{0}\right)-\check{\Pi}_{2}\left(t, \varpi_{0}\right)\right|,
\end{aligned}
$$

which shows that $\psi$ is a contraction. Thus, system (3) has a unique solution by Banach contraction theorem.

\section{Ulam-Hyres Stability}

In this section, we show that the numerical results are stable. Take a small change $\phi \in C[0, T]$, where $\phi(0)=0$ depends on the solution of $\psi\left(\widetilde{\Pi}\left(t, \varpi_{0}\right)\right)$ as

(i) For any positive $\varepsilon,|\phi(t)| \leq \varepsilon$ (ii) $D_{t}^{\gamma} \psi\left(\widetilde{\Pi}\left(t, \varpi_{0}\right)\right)=\Psi\left(t, \psi\left(\widetilde{\Pi}\left(t, \oplus_{0}\right)\right)+\phi(t)\right)$

Lemma 1. The above perturb problem has a solution,

$$
\begin{aligned}
D_{t}^{\gamma} \psi\left(\widetilde{\Pi}\left(t, \varpi_{0}\right)\right) & =\Psi\left(t, \psi\left(\widetilde{\Pi}\left(t, \varpi_{0}\right)\right)+\phi(t)\right), \\
\psi\left(\widetilde{\Pi}\left(0, \varpi_{0}\right)\right) & =\psi\left(\widetilde{\Pi}\left(0, \varpi_{0}\right)\right),
\end{aligned}
$$

if it satisfies

$$
\left|\left(\widetilde{\Pi}\left(t, \oplus_{0}\right)\right)-\left(\left(\widetilde{\Pi}(0)\left(t, \oplus_{0}\right)\right)+\frac{1}{\Gamma(\gamma)} \int_{0}^{t}(t-s)^{\gamma-1} \Psi(s,(\widetilde{\Pi}(t, s))) d s\right)\right| \leq \frac{T^{\gamma}}{\Gamma(\gamma+1)} \varepsilon=\Omega_{T, \alpha} \varepsilon .
$$

Proof. The proof is easy.

Theorem 4. Consider assumption (2) and relation (29) hold. Then, the numerical results of the considered model are
Ulam-Hyres stable if $\Delta=T^{\gamma} / \Gamma(\gamma+1) K_{\Psi}<1$, by showing that equation (25) is Ulam-Hyres stable.

Proof. Let $\widetilde{\Pi}\left(t, \omega_{0}\right) \in \mathscr{C}$ be any solution and $\widetilde{\widetilde{\Pi}}\left(t, \omega_{0}\right) \in \mathscr{C}$ be at most one solution of equation (25); then,

$$
\begin{aligned}
& \left|\widetilde{\Pi}\left(t, \varpi_{0}\right)-\overline{\widetilde{\Pi}}\left(t, \varpi_{0}\right)\right|=\left|\widetilde{\Pi}\left(t, \varpi_{0}\right)-\left(\widetilde{\Pi}_{0}\left(t, \varpi_{0}\right)+\frac{1}{\Gamma(\gamma)} \int_{0}^{t}(t-s)^{\gamma-1} \Psi\left(s, \widetilde{\widetilde{\Pi}}\left(s, \varpi_{0}\right)\right) d s\right)\right|, \\
& \leq\left|\widetilde{\Pi}\left(t, \oplus_{0}\right)-\left(\widetilde{\Pi}_{0}\left(t, \varpi_{0}\right)+\frac{1}{\Gamma(\gamma)} \int_{0}^{t}(t-s)^{\gamma-1} \Psi\left(s, \widetilde{\Pi}\left(s, \varpi_{0}\right)\right) d s\right)\right| \\
& +\left|\frac{1}{\Gamma(\gamma)} \int_{0}^{t}(t-s)^{\gamma-1} \Psi\left(s, \overline{\widetilde{\Pi}}\left(s, \varpi_{0}\right)\right) d s-\frac{1}{\Gamma(\gamma)} \int_{0}^{t}(t-s)^{\gamma-1} \Psi\left(s, \widetilde{\Pi}\left(s, \varpi_{0}\right)\right) d s\right|, \\
& \leq \Omega_{T, \gamma} \varepsilon+\Delta\left\|\widetilde{\Pi}\left(t, \varpi_{0}\right)-\overline{\widetilde{\Pi}}\left(t, \varpi_{0}\right)\right\| .
\end{aligned}
$$

From (29), we have

$$
\left\|\widetilde{\Pi}\left(t, \oplus_{0}\right)-\overline{\widetilde{\Pi}}\left(t, \oplus_{0}\right)\right\| \leq \frac{\Omega_{T, \gamma}}{1-\Delta} \varepsilon .
$$

Hence, from (30), it follows that system (6) is " $\mathrm{UH}$ stable." Consequently, the considered model is $\mathrm{UH}$ stable. 
4.1. Procedure for Solution. In this section, we deduce the solution of the given model by using LADM. Applying LT on (3), we obtain

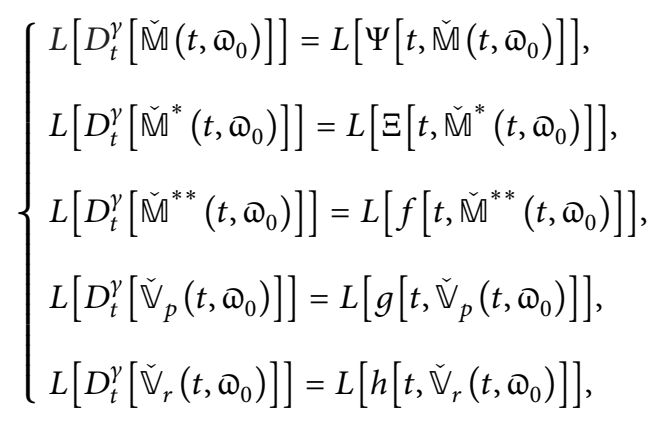

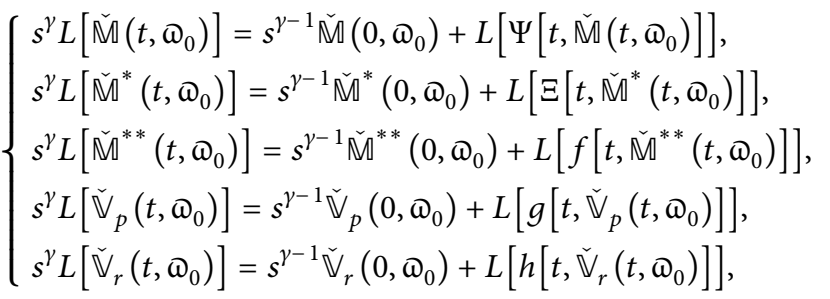

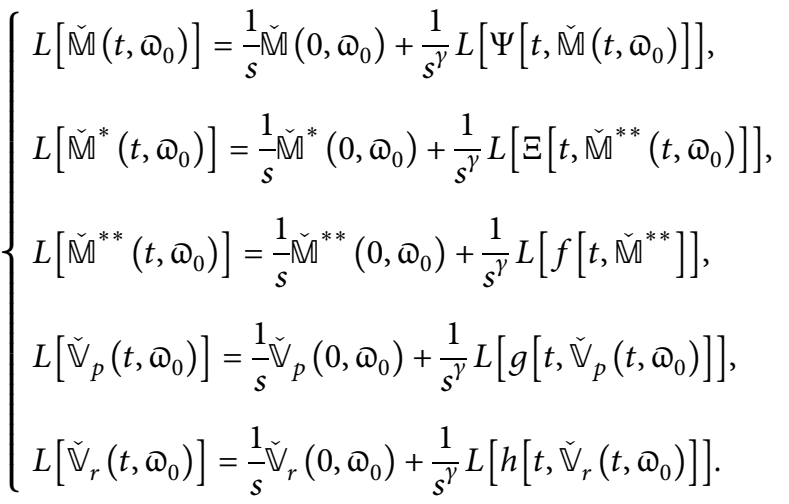

The infinite series solution is

$$
\begin{aligned}
& \check{\mathbb{M}}\left(t, \Phi_{0}\right)=\sum_{m=0}^{\infty} \check{\mathbb{M}}_{m}\left(t, \Phi_{0}\right), \check{\mathbb{M}}^{*}\left(t, \Phi_{0}\right)=\sum_{m=0}^{\infty} \check{M}_{m}^{*}\left(t, \Phi_{0}\right), \\
& \check{\mathbb{M}}^{* *}\left(t, \Phi_{0}\right)=\sum_{m=0}^{\infty} \check{\mathbb{M}}_{m}^{* *}\left(t, \Phi_{0}\right), \check{\mathbb{V}}_{p}\left(t, \varpi_{0}\right)=\sum_{m=0}^{\infty} \check{\mathbb{V}}_{p_{m}}\left(t, \Phi_{0}\right), \\
& \check{\mathbb{V}}_{r}\left(t, \oplus_{0}\right)=\sum_{m=0}^{\infty} \check{\mathbb{V}}_{r_{m}}\left(t, \oplus_{0}\right) \\
& \check{\mathbb{M}}\left(t, \Phi_{0}\right) \check{\mathbb{V}}_{p}\left(t, \Phi_{0}\right)=\sum_{n=0}^{\infty} \mathscr{Z}_{1, n} \check{\mathbb{V}}_{r}\left(t, \varpi_{0}\right) \check{M}^{*}\left(t, \Phi_{0}\right) \sum_{n=0}^{\infty} \mathscr{Z}_{2, n},
\end{aligned}
$$

where $\mathscr{Z}_{1, n}$ and $\mathscr{Z}_{2, n}$ are Adomian polynomials and represent nonlinear terms.

Taking inverse Laplace transform, we obtain

$$
\begin{aligned}
& \int \sum_{m=0}^{\infty} \check{\mathbb{M}}_{m}\left(t, \oplus_{0}\right)=\check{M}\left(0, \varpi_{0}\right)+L^{-1}\left[\frac{1}{s^{\gamma}} L\left[\Psi\left[t, \check{M}\left(t, \oplus_{0}\right)\right]\right]\right] \text {, } \\
& \sum_{m=0}^{\infty} \check{\mathbb{M}}_{m}^{*} t, \varpi_{0}=\check{\mathbb{M}}^{*}\left(0, \varpi_{0}\right)+L^{-1}\left[\frac{1}{s^{\gamma}} L\left[\Xi\left[t, \check{M}^{* *}\left(t, \varpi_{0}\right)\right]\right]\right], \\
& \left\{\sum_{m=0}^{\infty} \check{\mathbb{M}}_{m}^{* *}\left(t, \oplus_{0}\right)=\check{\mathbb{M}}^{* *}\left(0, \oplus_{0}\right)+L^{-1}\left[\frac{1}{s^{\gamma}} L\left[f\left[t, \check{\mathbb{M}}^{* *}\right]\right]\right]\right. \\
& \sum_{m=0}^{\infty} \check{\mathbb{V}}_{p_{m}}\left(t, \Phi_{0}\right)=\check{\mathbb{V}}_{p}\left(0, \varpi_{0}\right)+L^{-1}\left[\frac{1}{s^{\gamma}} L\left[g\left[t, \check{\mathbb{V}}_{p}\left(t, \varpi_{0}\right)\right]\right]\right], \\
& \sum_{m=0}^{\infty} \check{\mathbb{V}}_{r_{m}}\left(t, \oplus_{0}\right)=\check{\mathbb{V}}_{r}\left(0, \oplus_{0}\right)+L^{-1}\left[\frac{1}{s^{\gamma}} L\left[h\left[t, \check{\mathbb{V}}_{r}\left(t, \oplus_{0}\right)\right]\right]\right]
\end{aligned}
$$

Comparing the first two terms of the series in parametric form,

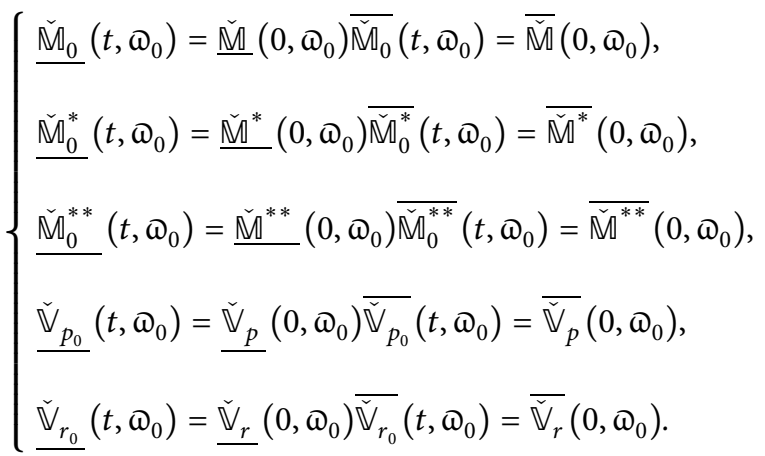

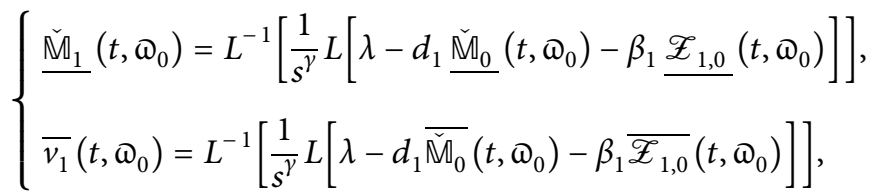




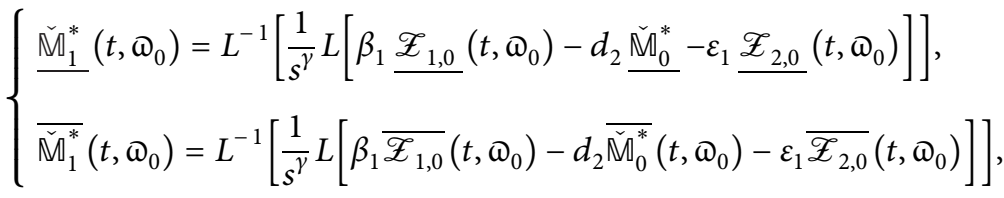

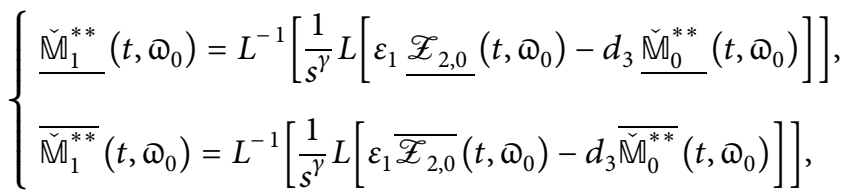

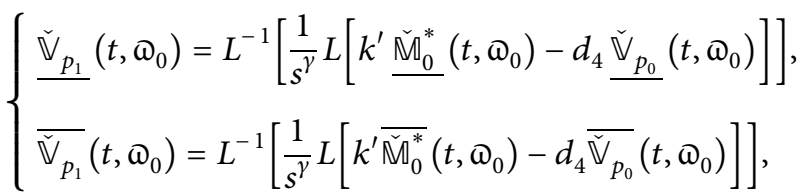

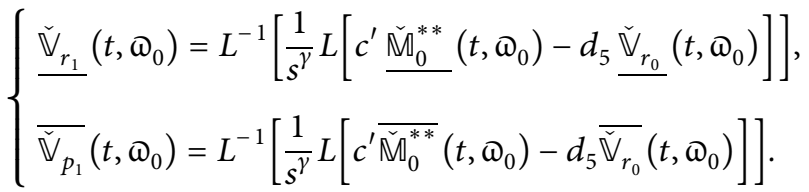

We can find other terms by the similar way. Hence, the system has the series solution as follows:

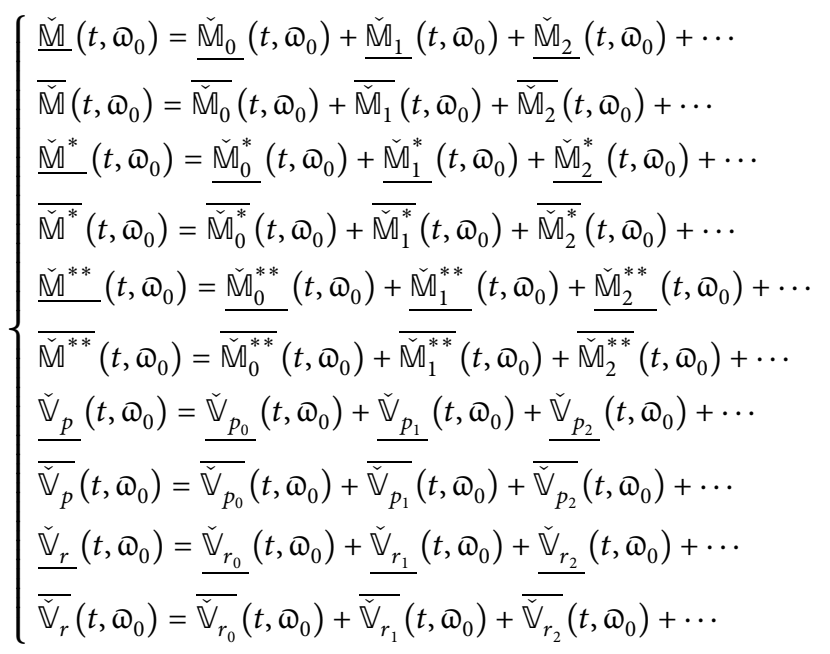

Based on the procedure of the method, the following theorem discusses the convergence of the proposed method.

Theorem 5. Let $H$ be a Banach space, $\widetilde{\vartheta}_{p}(\delta, t), \widetilde{\vartheta}_{n}(\delta, t) \in H$, and $0<\xi<1$. Let $\widetilde{\vartheta}(\delta, t)$ be an exact solution of the considered model. Then, the series solution $\sum_{p=0}^{\infty} \widetilde{\vartheta}_{p}(\delta, t)$ converges to the exact solution $\widetilde{\vartheta}(\delta, t)$ whenever $\widehat{\vartheta}_{p}(\delta, t) \leq \xi \widetilde{\vartheta}_{p-1}(\delta, t)$ for all
$p>\mathbb{T}$; i.e., for any $\omega>0$, there exists a positive number $\mathbb{T}$ such that $\left\|\widetilde{\vartheta}_{p+n}(\delta, t)\right\| \leq \omega, \forall m, n>\mathbb{T}$.

Proof. Let us define the sequence of $\sum_{p=0}^{\infty} \widetilde{\vartheta}_{p}(\delta, t)$ as

$$
\begin{aligned}
\mathbb{S}_{0}(\delta, t) & =\widetilde{\vartheta}_{0}(\delta, t), \\
\mathbb{S}_{1}(\delta, t) & =\widetilde{\vartheta}_{0}(\delta, t)+\widetilde{\vartheta}_{1}(\delta, t), \\
\mathbb{S}_{2}(\delta, t) & =\widetilde{\vartheta}_{0}(\delta, t)+\widetilde{\vartheta}_{1}(\delta, t)+\widetilde{\vartheta}_{2}(\delta, t), \\
\mathbb{S}_{3}(\delta, t) & =\widetilde{\vartheta}_{0}(\delta, t)+\widetilde{\vartheta}_{1}(\delta, t)+\widetilde{\vartheta}_{2}(\delta, t)+\widetilde{\vartheta}_{3}(\delta, t), \\
\vdots & \\
\mathbb{S}_{p}(\delta, t) & =\widetilde{\vartheta}_{0}(\delta, t)+\widetilde{\vartheta}_{1}(\delta, t)+\widetilde{\vartheta}_{2}(\delta, t)+\cdots+\widetilde{\vartheta}_{p}(\delta, t) .
\end{aligned}
$$

We need to show that $\mathbb{S}_{p}(\delta, t)$ is a Cauchy sequence in $H$. For this, we consider

$$
\begin{aligned}
\left\|\mathbb{S}_{p+1}(\delta, t)-\mathbb{S}_{p}(\delta, t)\right\| & =\left\|\widetilde{\vartheta}_{p+1}(\delta, t)\right\|, \\
& \leq \xi\left\|\widetilde{\vartheta}_{p}(\delta, t)\right\| \\
& \leq \xi^{2}\left\|\widetilde{\vartheta}_{p-1}(\delta, t)\right\| \\
& \leq \xi^{3}\left\|\widetilde{\vartheta}_{p-2}(\delta, t)\right\| \\
& \vdots \\
& \leq \xi^{p+1}\left\|\widetilde{\vartheta}_{0}(\delta, t)\right\| .
\end{aligned}
$$


Now, for any $p, n \in \mathbb{N}$, we have

$$
\begin{aligned}
\left\|\mathbb{S}_{p}(\delta, t)-\mathbb{S}_{n}(\delta, t)\right\|= & \left\|\widetilde{\mathfrak{\vartheta}}_{p+n}(\delta, t)\right\| \\
= & \left\|\left(\mathbb{S}_{p}(\delta, t)-\mathbb{S}_{p-1}(\delta, t)\right)+\left(\mathbb{S}_{p-1}(\delta, t)-\mathbb{S}_{p-2}(\delta, t)\right)+\left(\mathbb{S}_{p-2}(\delta, t)-\mathbb{S}_{p-3}(\delta, t)\right)+\cdots+\left(\mathbb{S}_{n+1}(\delta, t)-\mathbb{S}_{n}(\delta, t)\right)\right\| \\
\leq & \left\|\mathbb{S}_{p}(\delta, t)-\mathbb{S}_{p-1}(\delta, t)\right\|+\left\|\mathbb{S}_{p-1}(\delta, t)-\mathbb{S}_{p-2}(\delta, t)\right\| \\
& +\cdots+\left\|\mathbb{S}_{n+1}(\delta, t)-\mathbb{S}_{n}(\delta, t)\right\| . \\
\leq & \xi^{p}\left\|\widetilde{\mathfrak{\vartheta}}_{0}(\delta, t)\right\|+\xi^{p-1}\left\|\widetilde{\mathfrak{9}}_{0}(\delta, t)\right\|+\cdots+\xi^{p+1}\left\|\widetilde{\mathfrak{\vartheta}}_{0}(\delta, t)\right\| \\
= & \left\|\widetilde{\mathfrak{\vartheta}}_{0}(\delta, t)\right\|\left(\xi^{p}+\xi^{p-1}+\cdots+\xi^{p+1}\right) \\
= & \left\|\widetilde{\mathfrak{\vartheta}}_{0}(\delta, t)\right\| \frac{1-\xi^{p-n}}{1-\xi} \xi^{n+1} .
\end{aligned}
$$

Since $\xi \in(0,1)$ and $\widetilde{\vartheta}_{0}(\delta, t)$ is bounded, letting $\omega=1-\xi /\left(1-\xi^{p-n}\right) \xi^{n+1}\left\|\widetilde{\vartheta}_{0}(\delta, t)\right\|$, we obtain

$$
\left\|\widetilde{\vartheta}_{p+n}(\delta, t)\right\| \leq \omega, \forall p, n>\mathbb{T} \text {. }
$$

Thus, the sequence $\left\{\widetilde{\vartheta}_{p}(\delta, t)\right\}_{p=0}^{\infty}$ is a Cauchy sequence in $H$. This implies that $\exists \widetilde{\vartheta}(\delta, t) \in H$ such that $\lim _{p \longrightarrow \infty} \widetilde{\vartheta}_{p}(\delta, t)=\mathscr{\vartheta}(\delta, t)$. Thus, the required solution converges to the exact solution.

\section{Numerical Results and Discussion}

In this section, we illustrate the obtained solution of the proposed model through MATLAB-17. We have taken the parameter values from [7]. The values of parameters are $\lambda=2 \mathrm{cell} / \mathrm{mm}^{3}, d_{1}=0.01 /$ day $, \quad \beta_{1}=0.004 \mathrm{~mm}^{3} / \mathrm{vir}, d_{2}=$ $0.33 /$ day $, \epsilon_{1}=0.004 \mathrm{~mm}^{3} / \mathrm{vir}, d_{3}=2 /$ day,$k^{\prime}=50 \mathrm{vir} / \mathrm{cell}$, $d_{4}=2 /$ day,$c^{\prime}=2000$ vir $/$ cell , and $d_{5}=2 /$ day. Consider model (2) under initial conditions as

$$
\begin{aligned}
& \check{M}\left(0, \oplus_{0}\right)=\left(\oplus_{0}-1,1-\oplus_{0}\right), \\
& \check{M}^{*}\left(0, \varpi_{0}\right)=\left(\varpi_{0}-1,1-\varpi_{0}\right) \text {, } \\
& \check{M}^{* *}\left(0, \Phi_{0}\right)=\left(\varpi_{0}-1,1-\varpi_{0}\right) \text {, } \\
& \check{\mathbb{V}}_{p}\left(0, \oplus_{0}\right)=\left(\varpi_{0}-1,1-\varpi_{0}\right) \text {, } \\
& \check{\mathbb{V}}_{r}\left(0, \varpi_{0}\right)=\left(\varpi_{0}-1,1-\varpi_{0}\right) \text {, }
\end{aligned}
$$

where $\varpi_{0} \in[0,1]$. Using the developed technique on model (2) by applying the initial conditions, we obtain

$$
\begin{aligned}
& \underline{\check{M}_{0}}\left(t, \varpi_{0}\right)=\varpi_{0}-1, \overline{\mathbb{M}_{0}}\left(t, \varpi_{0}\right)=1-\varpi_{0}, \\
& \check{\mathbb{M}}_{0}^{*}\left(t, \Phi_{0}\right)=\Phi_{0}-1, \overline{\check{M}_{0}^{*}}\left(t, \Phi_{0}\right)=1-\Phi_{0}, \\
& \check{\mathbb{M}}_{0}^{* *}\left(t, \varpi_{0}\right)=\varpi_{0}-1, \check{\mathbb{M}}_{0}^{* *}\left(t, \varpi_{0}\right)=1-\Phi_{0}, \\
& \check{\mathbb{V}}_{p_{0}}\left(t, \varpi_{0}\right)=\varpi_{0}-1, \overline{\mathbb{V}}_{p_{0}}\left(t, \varpi_{0}\right)=1-\varpi_{0}, \\
& \underline{\check{\mathbb{V}}_{r_{0}}}\left(t, \oplus_{0}\right)=\Phi_{0}-1, \overline{\mathbb{V}}_{r_{0}}\left(t, \oplus_{0}\right)=1-\varpi_{0},
\end{aligned}
$$

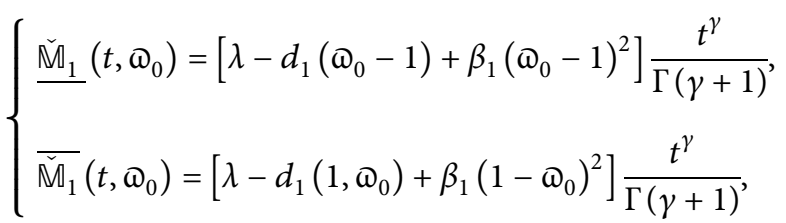

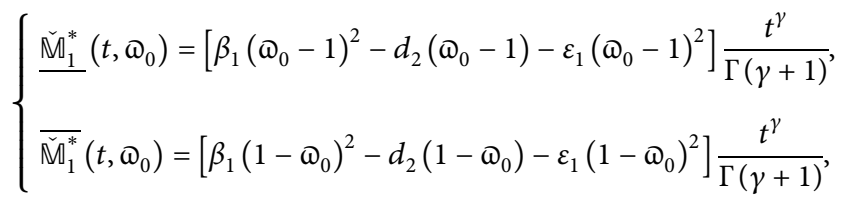

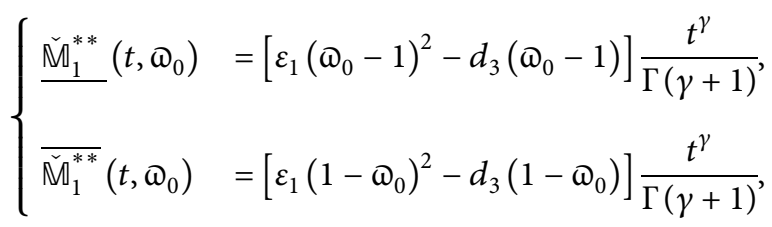




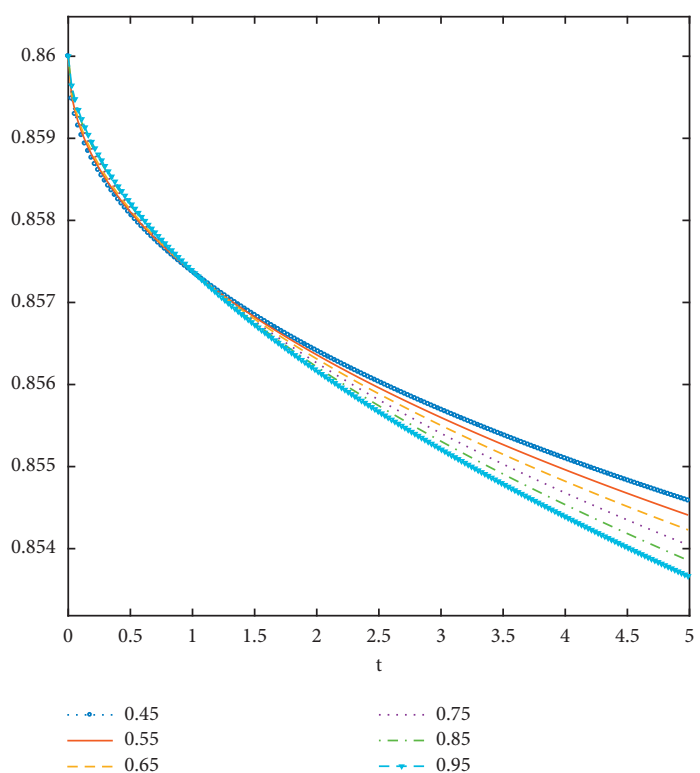

(a)

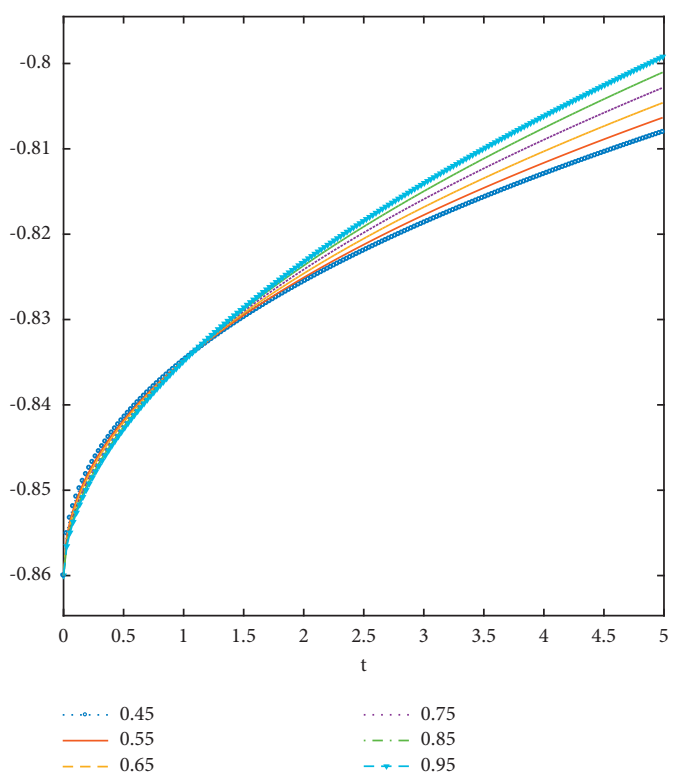

(b)

Figure 1: 2D simulation of $\check{M}\left(t, \oplus_{0}\right)$ at different fractional orders and $\oplus_{0}=0.95$. (a) Uppercase. (b) Lowercase.

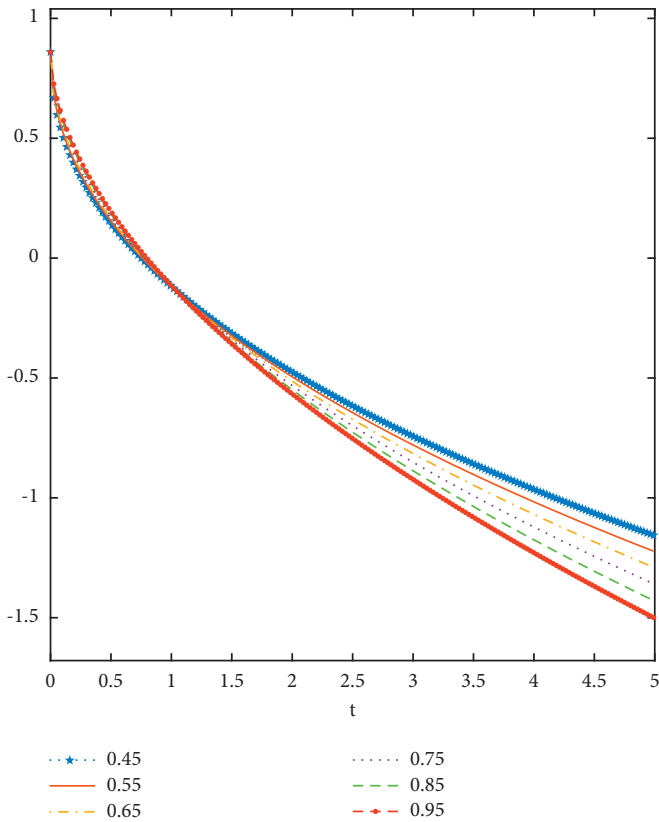

(a)

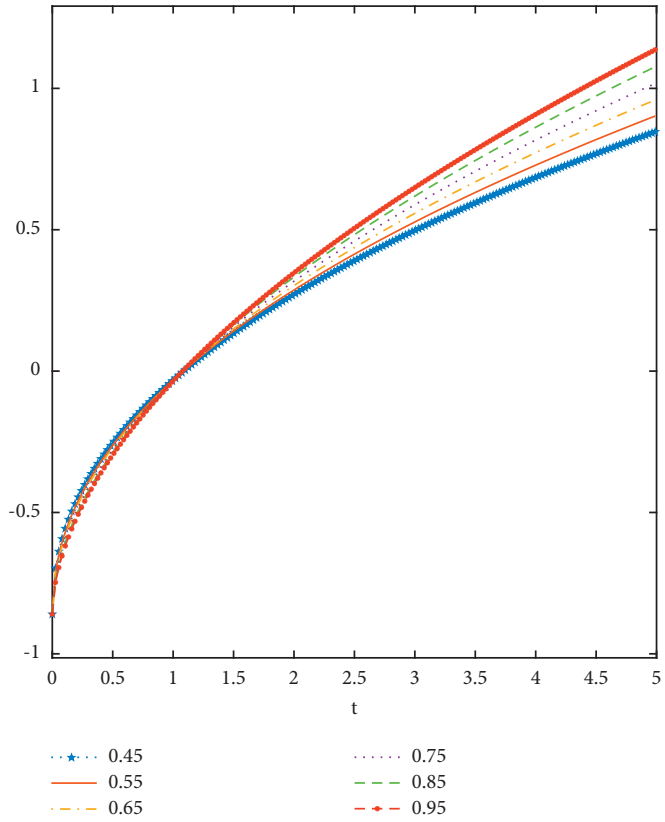

(b)

FIgURe 2: 2D simulation of $\check{\mathbb{M}}^{*}\left(t, \Phi_{0}\right)$ at different fractional orders and $\oplus_{0}=0.95$. (a) Uppercase. (b) Lowercase.

$$
\left\{\begin{array}{l}
\underline{\check{\mathbb{V}}_{p_{1}}}\left(t, \varpi_{0}\right)=\left[k^{\prime}\left(\varpi_{0}-1\right)-d_{4}\left(\varpi_{0}-1\right)\right] \frac{t^{\gamma}}{\Gamma(\gamma+1)} \\
\check{\mathbb{V}}_{p_{1}}\left(t, \varpi_{0}\right)=\left[k^{\prime}\left(1-\varpi_{0}\right)-d_{4}\left(1-\varpi_{0}\right)\right] \frac{t^{\gamma}}{\Gamma(\gamma+1)}
\end{array}\right.
$$

$$
\left\{\begin{array}{l}
\check{\mathbb{V}}_{r_{1}}\left(t, \Phi_{0}\right)=\left[c^{\prime}\left(\omega_{0}-1\right)-d_{5}\left(\Phi_{0}-1\right)\right] \frac{t^{\gamma}}{\Gamma(\gamma+1)}, \\
\check{\mathbb{V}}_{r_{1}}\left(t, \omega_{0}\right)=\left[c^{\prime}\left(1-\omega_{0}\right)-d_{5}\left(1-\omega_{0}\right)\right] \frac{t^{\gamma}}{\Gamma(\gamma+1)} .
\end{array}\right.
$$




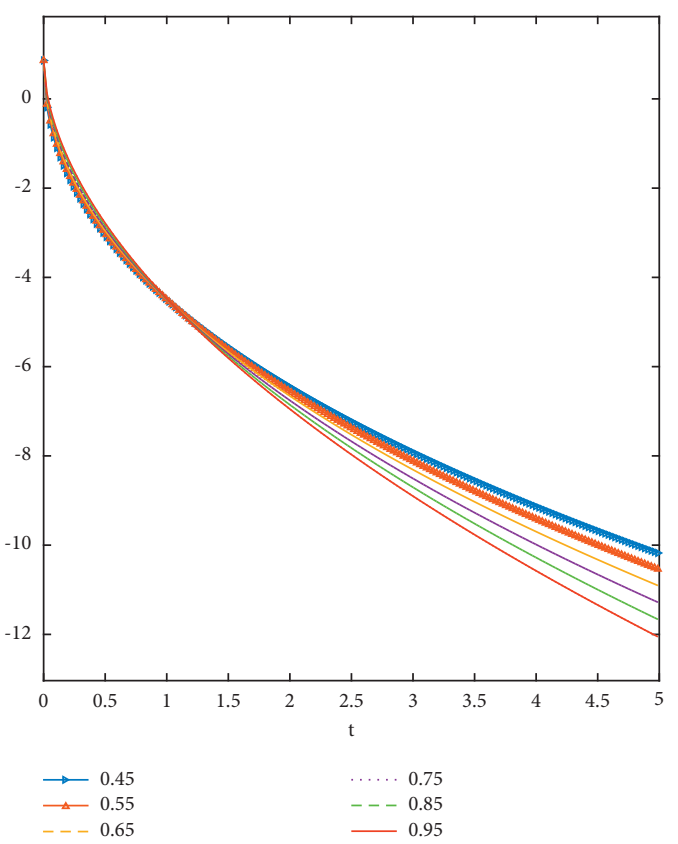

(a)

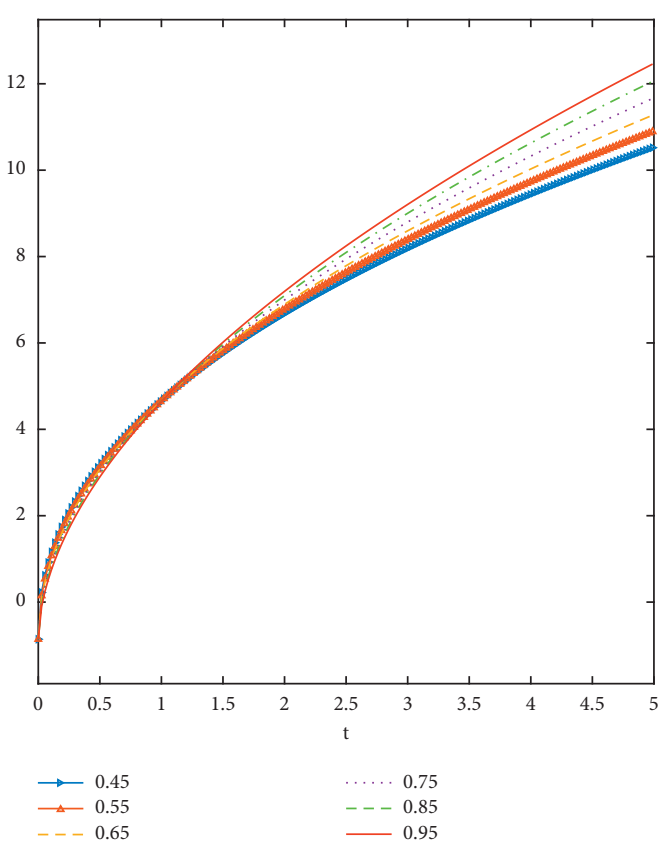

(b)

FIgURE 3: $2 \mathrm{D}$ simulation of $\check{M}^{* *}\left(t, \varpi_{0}\right)$ at different fractional orders and $\varpi_{0}=0.95$. (a) Uppercase. (b) Lowercase.

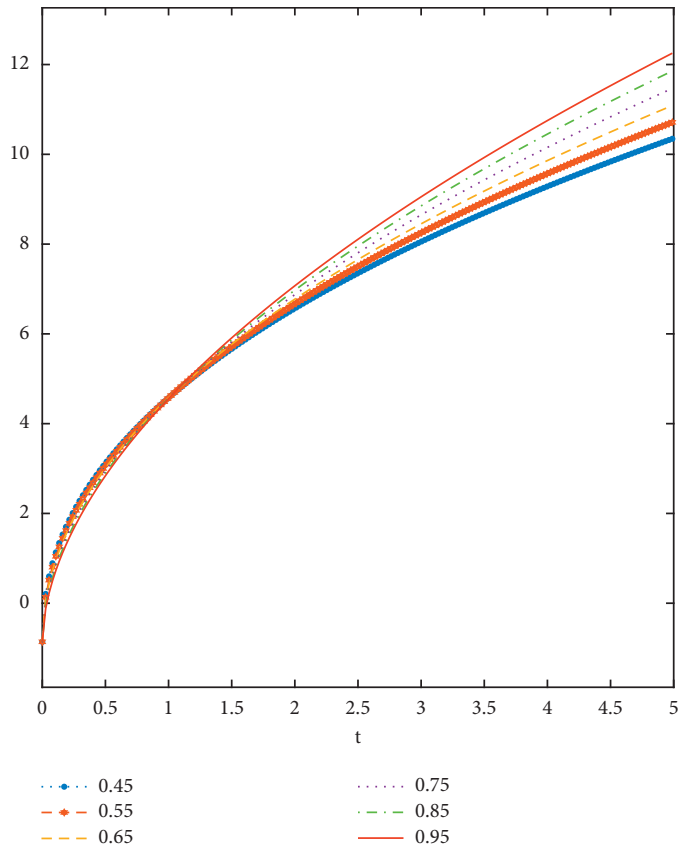

(a)

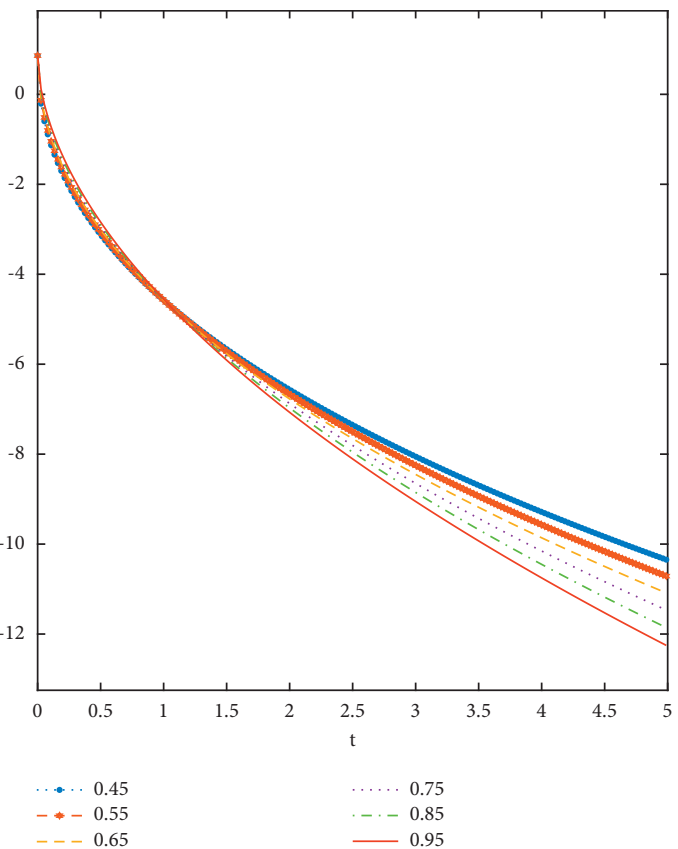

(b)

Figure 4: $2 \mathrm{D}$ simulation of $\mathbb{V}_{p}\left(t, \varpi_{0}\right)$ at different fractional orders and $\varpi_{0}=0.95$. (a) Uppercase. (b) Lowercase.

Other terms can be found out by the similar way.

We have simulated the numerical results obtained for the different compartments via Matlab. From the literature, it is clear the fractional operators have a greater degree of freedom. Therefore, we have taken only a few orders for the graphical representation. Each class has a lower and upper solution and has presented at different fractional orders, where the uncertainty lies in $[0,1]$. The odd-numbered Figures 1-5 represent two-dimensional graphs for the various compartments at various fractional orders. While 


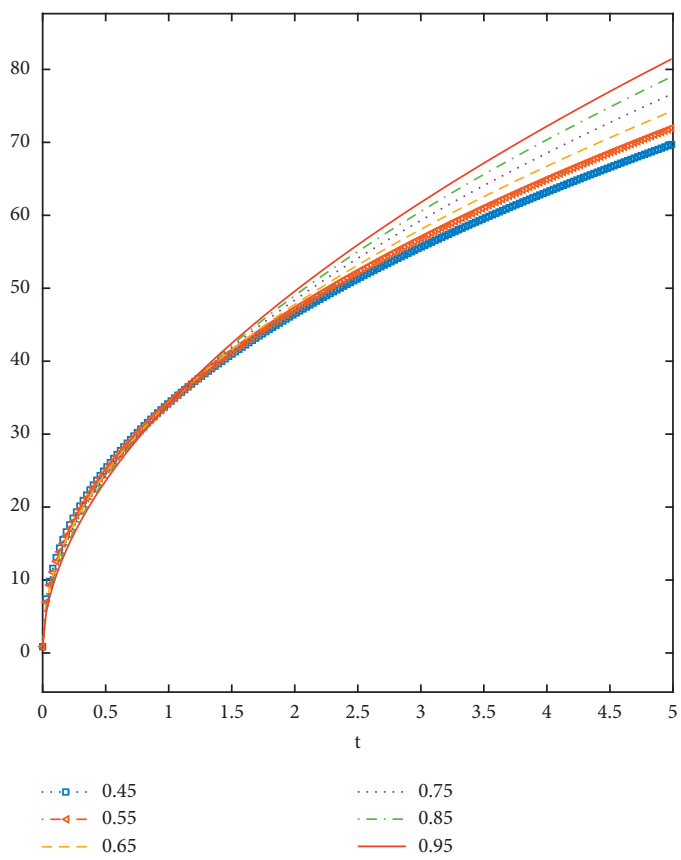

(a)

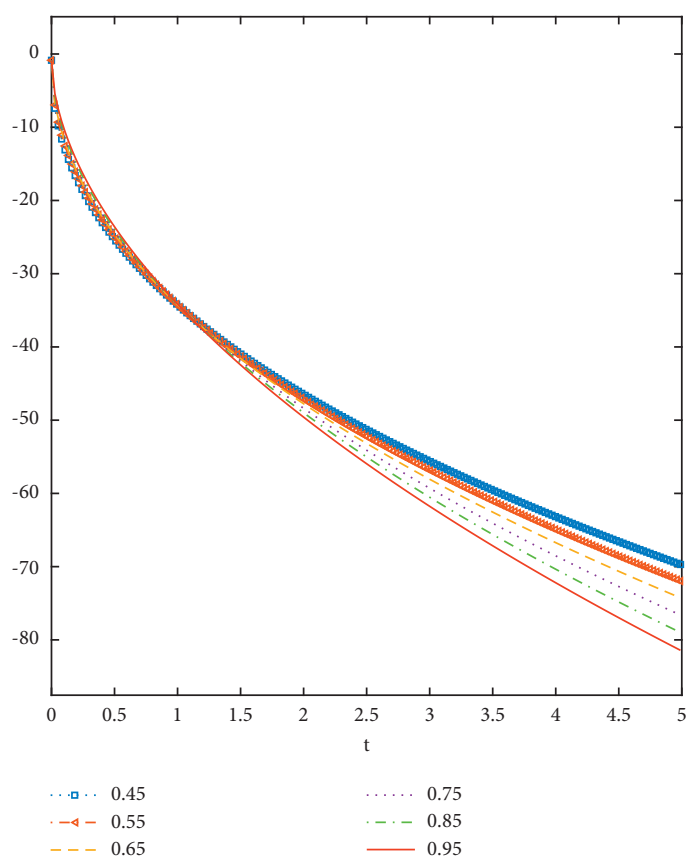

(b)

Figure 5: $2 \mathrm{D}$ simulation of $\mathbb{V}_{r}\left(t, \varpi_{0}\right)$ at different fractional orders and $\varpi_{0}=0.95$. (a) Uppercase. (b) Lowercase.
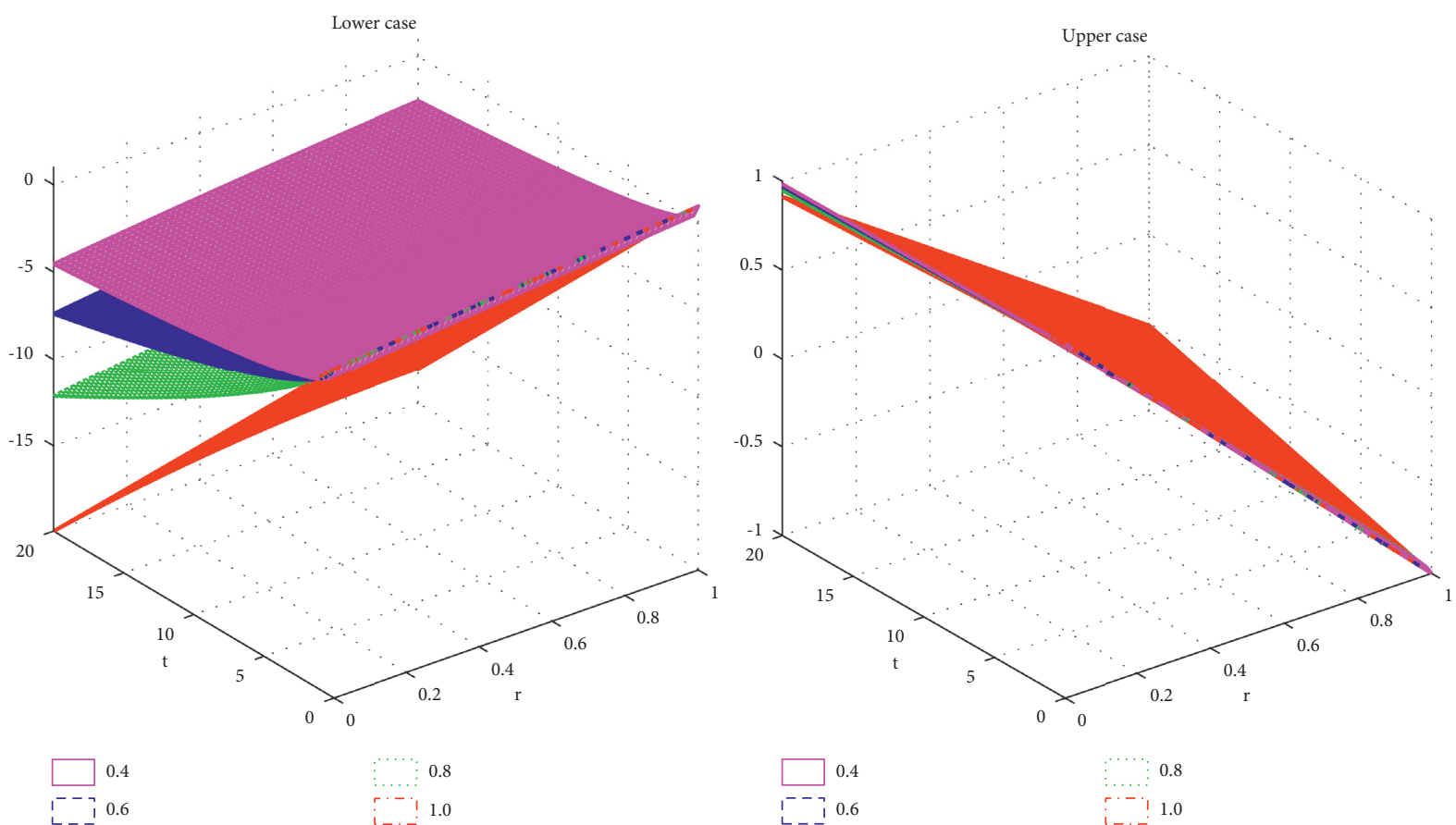

$\underset{\square}{\square} 0.4$

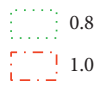

10.6

FIgURE 6: 3D simulation of $\check{M}\left(t, \varpi_{0}\right)$ at different fractional orders and $\varpi_{0} \in[0,1]$.

the even-numbered Figures 6-10 represent three-dimensional graphs of the different classes at various fractional orders and uncertainty belongs to $[0,1]$. The fractional order has a great impact on the lower and upper solution of the considered model. As the fractional order increases from lower value to higher values, the increase in the uppercase and decrease in lowercase become faster. The solution curves of will converge to integer-order solution when the fractional order tends to unity. Thus, the considered model (2) is generalized than model (1). 

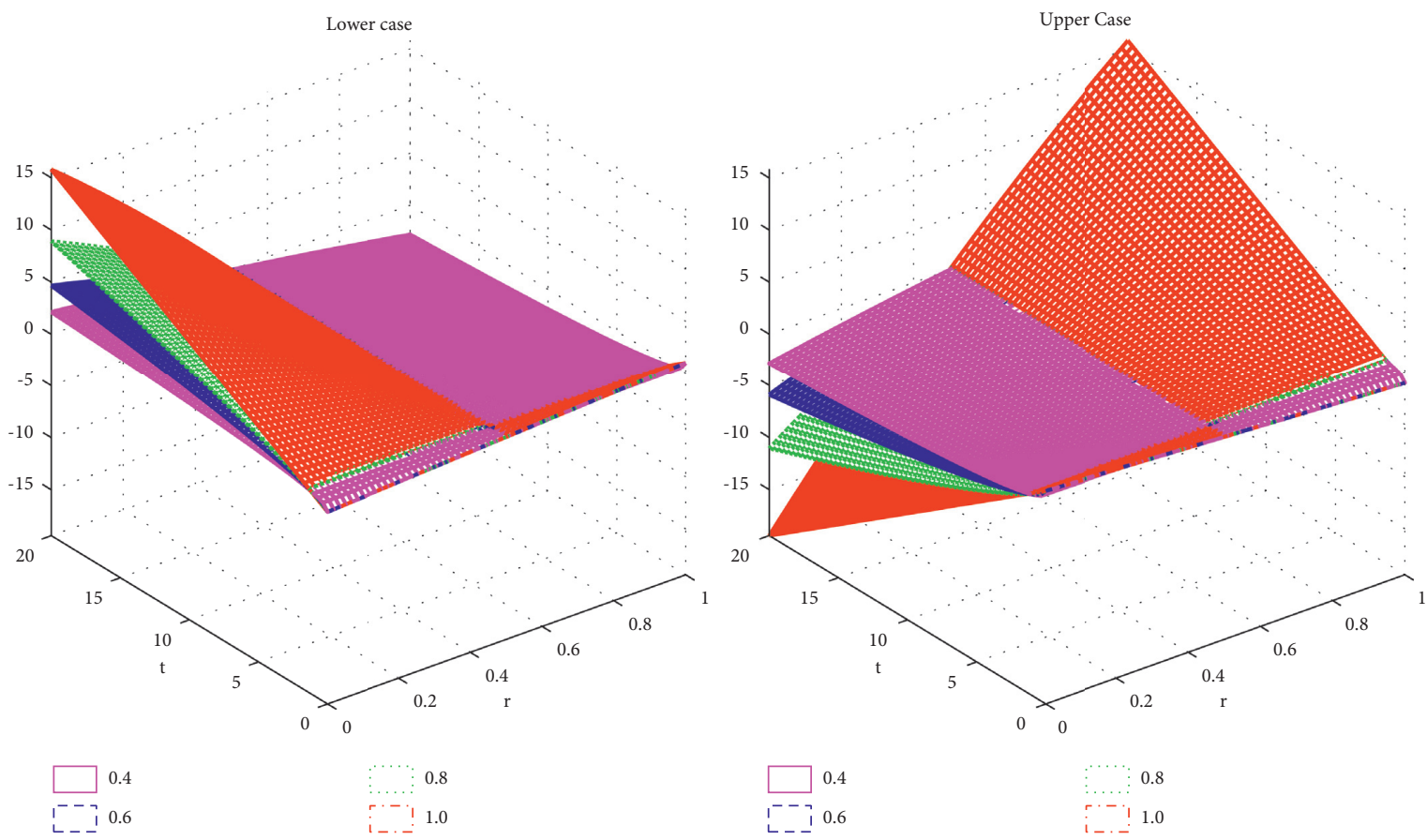

FIgURE 7: 3D simulation of $\check{M}^{*}\left(t, \Phi_{0}\right)$ at different fractional orders and $\varpi_{0} \in[0,1]$.
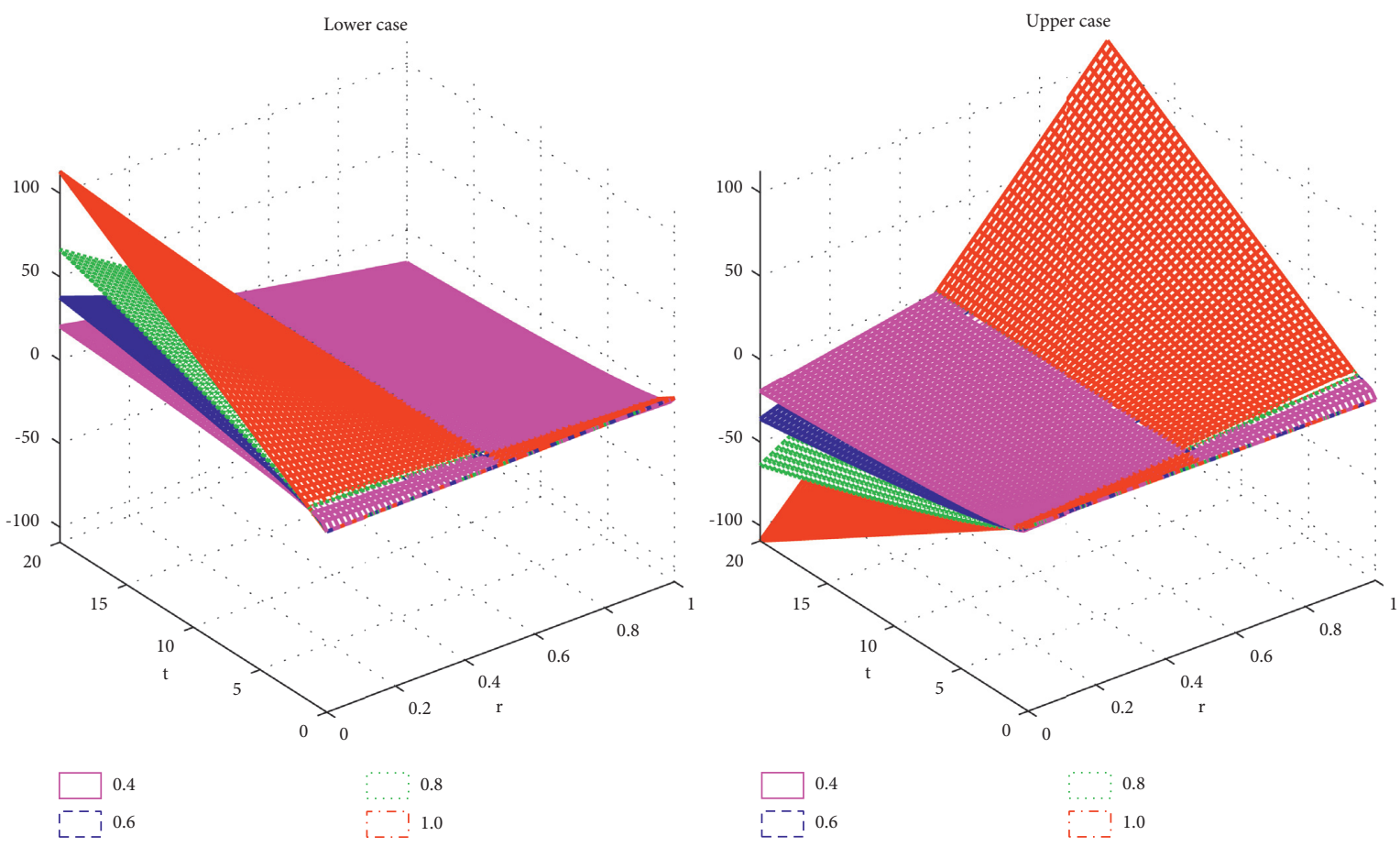

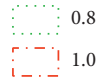

Figure 8: 3D simulation of $\check{M}^{* *}\left(t, \varpi_{0}\right)$ at different fractional orders and $\varpi_{0} \in[0,1]$. 

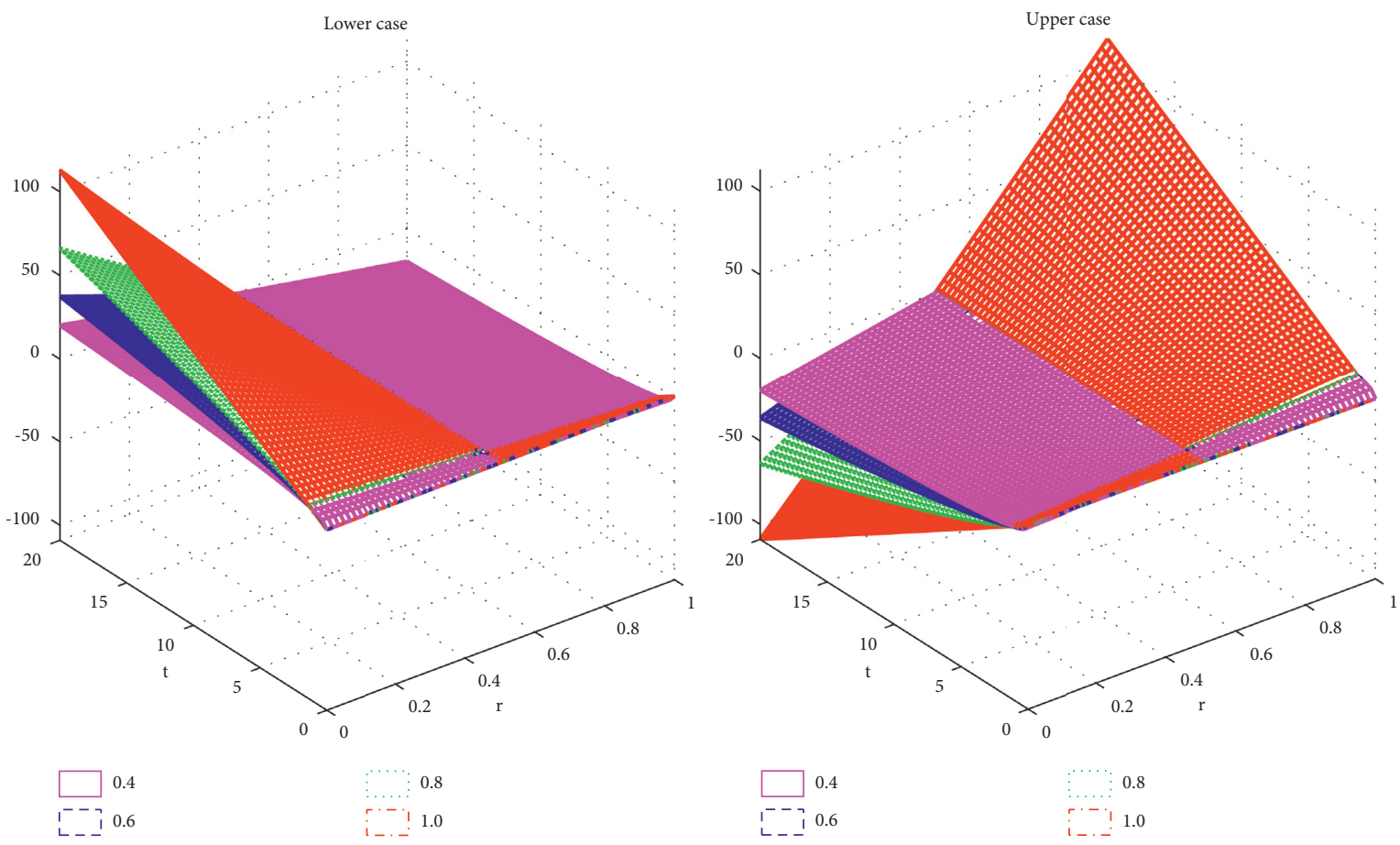

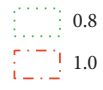

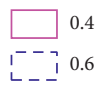

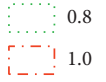

FIgURE 9: $3 D$ simulation of $\mathbb{V}_{p}\left(t, \varpi_{0}\right)$ at different fractional orders and $\varpi_{0} \in[0,1]$.
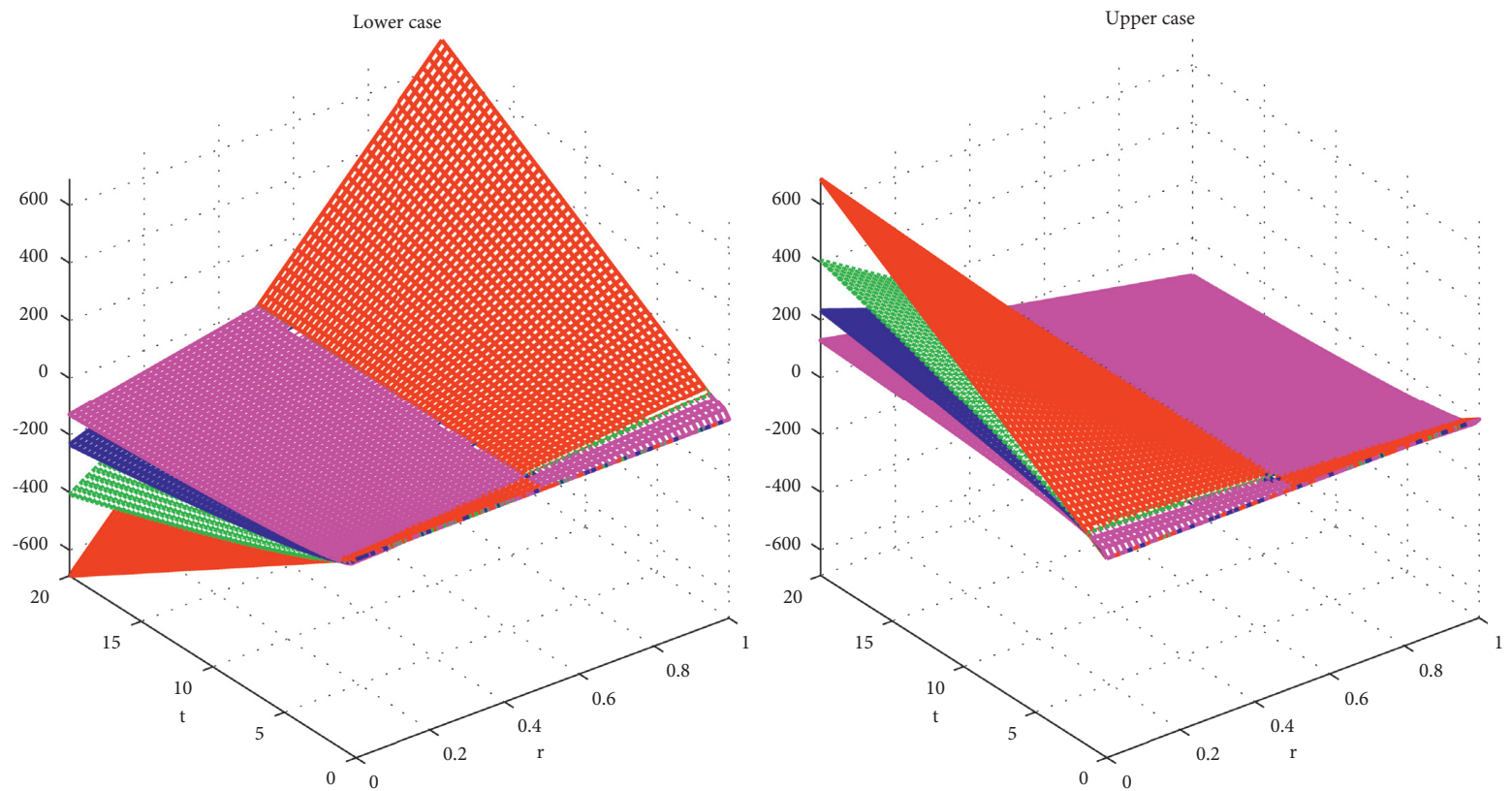

$\square 0.4$
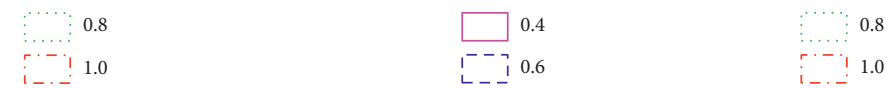

Figure 10: $3 \mathrm{D}$ simulation of $\mathbb{V}_{r}\left(t, \Phi_{0}\right)$ at different fractional orders and $\Phi_{0} \in[0,1]$. 


\section{Conclusion}

We have successfully extended the fractional-order model of HIV to the fuzzy fractional model under the Caputo operator. We have derived the existence and uniqueness results through fixed-point theorems. We have also deduced the Ulam-Hyres stability of the proposed model through nonlinear analysis. We have presented a general method via the fuzzy LADM to obtain an approximate solution of the proposed model. We have achieved the numerical results for the specific fuzzy initial conditions through the developed scheme. We have provided the numerical simulations of the numerical results at different uncertainty values and different fractional-order. The simulations show that fuzziness with fractional operators provides global dynamics of a physical problem. Thus, these concepts open new doors for young researchers to model any physical problem with fuzzy fractional operators. The considered model can be studied under more generalized operators in the fuzzy case.

\section{Data Availability}

The data used support the findings of this study are available from the corresponding author upon request.

\section{Conflicts of Interest}

The authors have no conflicts of interest regarding this article.

\section{References}

[1] A. A. Okoye and L. J. Picker, "CD4+T-cell depletion in HIV infection: mechanisms of immunological failure," Immunological Reviews, vol. 254, no. 1, pp. 54-64, 2013.

[2] R. A. Arnaout, N. Martin, and D. Wodarz, "HIV-1 dynamics revisited: biphasic decay by cytotoxic T lymphocyte killing?" Proceedings of the Royal Society of London-Series B: Biological Sciences, vol. 267, no. 1450, pp. 1347-1354, 2000.

[3] B. S. Chan and P. Yu, "Bifurcation analysis in a model of cytotoxic T-lymphocyte response to viral infections," Nonlinear Analysis: Real World Applications, vol. 13, no. 1, pp. 64-77, 2012.

[4] M. Ghoreishi, A. I. B. M. Ismail, and A. K. Alomari, “Application of the homotopy analysis method for solving a model for HIV infection of CD4+ T-cells," Mathematical and Computer Modelling, vol. 54, no. 11-12, pp. 3007-3015, 2011.

[5] M. Y. Ongun, "The Laplace Adomian decomposition method for solving a model for HIV infection of CD4+T cells," Mathematical and Computer Modelling, vol. 53, no. 5-6, pp. 597-603, 2011.

[6] M. Merdan, "Homotopy perturbation method for solving a model for HIV infection of CD4+ Tcells," Istanbul Ticaret Universitesi Fen Bilimleri Dergisi, vol. 12, no. 6, pp. 39-52, 2007.

[7] T. Revilla and G. Garćia-Ramos, "Fighting a virus with a virus: a dynamic model for HIV-1 therapy," Mathematical Biosciences, vol. 185, no. 2, pp. 191-203, 2003.

[8] S. Jesus, J. A. Tenreiro Machado, and J. Boaventure Cunha, "Fractional electrical impedances in botanical elements,"
Journal of Vibration and Control, vol. 14, no. 9-10, pp. 1389-1402, 2008.

[9] K. S. Miller and B. Ross, An Introduction to the Fractional Calculus and Fractional Differential Equations, A WileyInterscience Publication, JohnWiley \& Sons, New York, NY, USA, 1993.

[10] V. Lakshmikantham and A. S. Vatsala, "Basic theory of fractional differential equations," Nonlinear Anal, vol. 69, no. 8, pp. 2682-2677, 2008.

[11] M. Benchohra, A. Cabada, and D. Seba, "An existence result for nonlinear fractional differential equations on Banach spaces," Boundary Value Problems, p. 628916, 2009.

[12] N. Ali, K. Shah, and R. A. Khan, "Existence of positive solution to a class of fractional differential equations with three point boundary conditions," Mathematical Sciences Letters, vol. 5, no. 3, pp. 291-296, 2016.

[13] R. A. Khan and K. Shah, "Existence and uniqueness of solutions to fractional order multi- point boundary value problems," Communications in Applied Analysis, vol. 19, pp. 515-526, 2015.

[14] N. H. Sweilam, S. M. Al-Mekhlafi, Z. N. Mohammed, and D. Baleanu, "Optimal control for variable order fractional HIV/AIDS and malaria mathematical models with multi-time delay," Alexandria Engineering Journal, vol. 59, no. 5, pp. 3149-3162, 2020.

[15] N. H. Sweilam and S. M. Al-Mekhlafi, "Legendre spectralcollocation method for solving fractional optimal control of HIV infection of CD4+T cells mathematical model," The Journal of Defense Modeling and Simulation: Applications, Methodology, Technology, vol. 14, no. 3, pp. 273-284, 2017.

[16] N. H. Sweilam, W. A. Kareem, S. M. Al-Mekhlafi, and Z. Nabih, "Numerical treatments for a complex order fractional HIV infection model with drug resistance during therapy," Progr. Fract. Differ. Appl.vol. 7, no. 3, pp. 163-176, 2021.

[17] S. Saifullah, A. Ali, M. Irfan, and K. Shah, "Time-fractional klein-gordon equation with solitary/shock waves solutions," Mathematical Problems in Engineering, vol. 2021, no. 5, p. 15, 2021.

[18] S. Ahmad, A. Ullah, A. Akgül, and D. Baleanu, "Analysis of the fractional tumour-immune-vitamins model with MittagLeffler kernel," Results in Physics, vol. 19, p. 103559, 2020.

[19] S. Ahmad, A. Ullah, K. Shah, and A. Akgül, "Computational analysis of the third order dispersive fractional PDE under exponential-decay and Mittag-Leffler type kernels," Numerical Methods for Partial Differential Equations, pp. 1-16, 2020.

[20] M. Ishteva, Properties and Applications of the Caputo Fractional Operator, $\mathrm{PhD}$ thesis, Msc. thesis, Department of Mathematics, Universität Karlsruhe (TH), Sofia, Bulgaria, 2005.

[21] W. M. Abd-Elhameed and Y. H. Youssri, "Generalized Lucas polynomial sequence approach for fractional differential equations," Nonlinear Dynamics, vol. 89, no. 2, pp. 1341-1355, 2017.

[22] L. A. Zadeh, "Fuzzy sets," Information and Control, vol. 5, no. 1, pp. 338-353, 1965.

[23] S. S. L. Chang and L. A. Zadeh, "On fuzzy mapping and control," IEEE Transactions on Systems, Man, and Cybernetics, vol. SMC-2, no. 1, pp. 30-34, 1972.

[24] O. Kaleva, "Fuzzy differential equations," Fuzzy Sets and Systems, vol. 24, no. 3, pp. 301-317, 1987.

[25] S. Arshad and V. Luplescu, "Fractional differential equation with fuzzy initial conditon," The Electronic Journal of Differential Equations, vol. 34, pp. 1-8, 2011. 
[26] S. Ahmad, A. Ullah, K. Shah, S. Salahshour, A. Ahmadian, and T. Ciano, "Fuzzy fractional-order model of the novel coronavirus," Advances in Difference Equations, vol. 2020, no. 1, p. $472,2020$.

[27] S. Ahmad, A. Ullah, A. Ullah, A. Akgül, and T. Abdeljawad, "Computational analysis of fuzzy fractional order non-dimensional Fisher equation," Physica Scripta, vol. 96, p. 84004, 2021.

[28] A. Ullah, A. Ullah, S. Ahmad, I. Ahmad, and A. Akgül, "On solutions of fuzzy fractional order complex population dynamical model," Numerical Methods for Partial Differential Equations, pp. 1-21, 2020.

[29] H. J. Zimmermann, Fuzzy Set Theory and its Applications, Kluwer Academic Publishers, Dordrecht, 1991.

[30] L. A. Zadeh, "Fuzzy sets," Information and Control, vol. 8, no. 3, pp. 338-353, 1965.

[31] S. Salahshour, T. Allahviranloo, and S. Abbasbandy, "Solving fuzzy fractional differential equations by fuzzy Laplace transforms," Communications in Nonlinear Science and Numerical Simulation, vol. 17, no. 3, pp. 1372-1381, 2012.

[32] T. Allahviranloo, "Fuzzy fractional operators," Fuzzy Fractional Differential Operators and Equations, vol. 397, pp. 73-126, 2021. 